\title{
High-Temperature Plasma Physics
}

PPPL -2509

\author{
H.P. Furth \\ Princeton Plasma Physics Laboratory \\ Princeton, New Jersey 08543
}

DE38 009345

\section{Abstract}

Both magnetic and inertial confinement research a $=$ entering the plasma parameter range of fusion reactc interest. This paper reviews the individual and comno. technical problems of these two approaches to the generation of thermonuclear plasmas, and describes some related applications of high-temperature plasma physics.

\section{Introduction}

The basic theoretical principles of plasma physies were devel ped during the period 1930-1950. The temperatures of experimentally availa.jle plasmas, however, were generally not much above the first-ionization ene: $y$ range. Within this "low-temperature" regime, many practical applications of plasma physics were found in such fields as illumination, electrical switching, and the understanding of the ionosphere. During the 1950's, there emerged a highly ambitious new application: the controlled release of fusion energy from the nuclei of heavy hydrogen. The pursuit of this goal shifted the relevant plasma temperature from the atomic to the nuclear energy scale, and brought about the development of modern plasma physics.

The comprehensive density-temperature diagram for plasma physies ${ }^{1}$ is shown in Fig. 1. Hydrogen plasmas are well ionized above a temperature of about $1 \mathrm{eV}$ or $-10^{4}{ }^{\circ} \mathrm{K}$. The "ideal classical plasma" regime is defined as having a temperature that exceeds both the Coulomb interaction potential and the Fermi energy. The first condition implies the predominance of collective particle interactions through macroscopic electromagnetic fields (or equivalently, the presence of a large number of particles $n \lambda_{D}{ }^{3}$ within a Debye sphere). The second condition insures that quantum-med effects are generally smali. 
On this familiar $\mathrm{n}-\mathrm{T}$ diagram, blank spaces have traditionally been reserved for two projected fusion plasma regimes: (1) magnetically confined plasmas, with densities of $10^{14}-10^{15} \mathrm{~cm}^{-3}$ and temperatures of 10-30 keV, approaching the "relativistic plasma" border; (2) inertially confined plasmas with similar temperatures and with densities approaching the border marked "degenerate quantum plasmas." During the last several years, real experimental data have begun to appear in these reserved spaces. Two illustrative points are shown in Fig. 1. The corresponding state of progress towards the fusion energy objective, as measured by the product of central ion temperature, central density, and energy confinement time $\left(T_{i} n \tau_{E}\right)$, is indicated in Table $I$.

Experimental work on magnetically confined high-temperature plasmas began in earnest during the mid-50's, with inertial confinement experiments following about fifteen years later. These two flelds of research have gone through a notably similar pattern: Initial theoretical inspiration has been followed by a phase of experinental veration and anomaly that forced the growth of complex new physical understanding, yet has ultimately left a clear path to the fusion energy goal.

In the case of magnetic confinement, the a priori guidelines consisted mainly of ideal magnetohydrodynamics (MHD) and simple "classical" collisional transport theory. The predictions of MHD instability theory proved to be fairly relevant, but had to be enlarged in the direction of increased pessimism by allowance for finite electrical resistivity. In addition, large new classes of microinstabilities were discovered which were shown to result from non-thermal velocity distributions. In a high-temperature plasma, even the mild departures from the Maxwellian that are implied by the presence of spatial gradients in plasma density and 
temperature proved to be ample for the excitation of significant levels of microturbulence, along with transport rates well in excess of classical expectation.

The seeming failure of the initial theoretical visions in magnetic confinement research was aggravated by various imperfections of the real experimental world, such as geometric asynmetries and residual "atomic effects" due to incomplete ionization. With gradual advances in technology and increases in plasma size and magnetic field strength, these disturbing phenomena have been pushed out toward the edge of the main plasma (where they continue to exert a surprisingly strong effect on the quality of global energy confinement). As a result of better implementation, the performance of present-day devices is actually approaching initial theoretical expectation.

In the case of inertial confinement, the main a priori guidelines were provided by ordinary fluid dynamies, including elassical collisional transport and radiation-absorption. Somewhat analogous to the magnetic confinement experience, the main experimental dfficulties were associated with plasma gradients, edge effects, and imperfections: I-tense laser light was found to give rise to a varlety of harmful nonlinear plasna-wave interactions in the low-density corona surrounding the fuel pellets. The associated generation of non-Maxwellian energetic-electron components gave rise to "anomalous" heat propagation, preventing efficient pellet compression. Insufficiently uniform illumination of laser targets nelped drive Rayleigh-Taylor instabilities to unacceptable levels during the target-implosion phase. The physical understanding of these phenomena was expedited by contributions from theorists who had analyzed similar problems in the context of magnetically confired plasmas. 
As in the magnetic confinement case, inertial confinement experimenters soon discovered effective remedies: The use of shorter wave (visible and near-ultraviolet) laser light produced a marked reduction in collective phenomena at the plasma edge. "Indirect illumination" of targets has recently permitted astonishing target-compression factors to be achieved in spite of the Rayleigh-Taylor mode. Again, as in the magnetic confinement case, there is an unfortunate correlation between the direction of most effective experimental parameters (higher magnetic field strength/shorter laser wavelength) and the direction of greater technological cost and difficulty.

Ten years ago, when the author of the present paper was asked by the Department of Energy for a short definition of "the scientific feasibility of fusion power," he suggested: "Scientific feasibility is when they stop saying you can't do it and start saying they may not want it." According to this definition, both the magnetic and inertial confinement approaches are reaching the phase of scientific feasibility. There is no longer much serious doubt that ignited fusion plasmas can be achieved in laboratory experiments of affordable magnitude. The central issue has now become the economic viability of fusion power: the projected capital investment per MW of generating capacity, the minimum power rating per reactor unit, the estimated fractional availability at full power output, and the degree of environmental attractiveness relative to the two competing "inexhaustible" power sources, fission and solar power.

The pursuit of maximum economic potential tends to drive both magnetic and inertial fusion research into directions that depart somewhat from the proven lines of least experimental resistance. In the magnetic case, the two areas of most intensive research are the enhancement of the B-value 
(the ratio of plasma pressure $\mathrm{nT}$ to magnetic pressure $\mathrm{B}^{2} / 8 \pi$ ) and the provision of true steady-state plasma confinement. The objectives are to generate higher fusion power density, (proportional to $B^{2} B^{4}$ ) and to avoid the auxiliary costs and mechanical fatigue problems associated with a nonconstant heat source. The most successful experimental approach to date, the "tokamak" toroidal-confinement system has the potential to satisfy both these objectives by introducing varlous auxiliary features. There are also a number of alternative magnetic confinement solutions with inherently greater potential for high-B operation or true steady-state operation -- but with still unproven capabilities in the area of plasma confinement. The unpredictability of ultimate potentials on the plasma physics side of magnetic fusion research has lately been matched by the emergence of entirely new prospects on the side of magnet technology. Depending on the manufacturability of mechanically reliable magnet coils from the new high-temperature superconducting materials, the magnetic field strength of steady-state fusion reactors may rise significantly. The implied increase of fusion power density could turn out to satisfy the economic requirements even at present-day levels of the plasma B-value.

In the inertial confinement case, the main challenges are to minimize the energy input required to ignite a high-gain target, and to develop an efficient target-driver system, using practical high-power technology. Lower energy inputs imply smaller and more frequent target explosions, thus alleviating mechanical problems and minimizing the unit reactor power rating and cost. The currently "front-running" experimental approach, using X-rays from a laser-produced plasma for indirect illumination of targets, could be adapted in a number of ways to the economic requirements: One could retain indirect illumination, while seeking to 
improve its efficiency -- for instance, by replacing the laser-light oriver system with an energetic-ion driver. Alternatively, if the problem of uniform direct target illumination can be overcome by special optical techniques, the input energy requirement for ignition would be lowered, and the use of laser-driver technology could become more attractive for a practical reactor. A convenient improvement for all inertial (and magnetic) D-T fusion systems would be to spin-polarize the nuclei of the fuel, thus enhancing the D-T fusion-reaction rate in the plasma phase by a factor of $3 / 2$. Polarized fuel could have a particularly great benefit in reducing the ignition-energy requirement for inertial fusion.

In addition to the special technological problems that are inherent in their individual approaches, the magnetic and inertial fusion research prograns face some common issues in the area of nuclear technology. The 14-MeV neutron and the $3.5-\mathrm{MeV}$ a-particle of the D-T reaction represent very "high-grade" energy -- but the exploitation of this energy source with present-day fission-reactor technology is problematical. Since a-particles are electrically charged, they are useful for self-heating (or "igniting") the fusion fuel, which is important for the achievement of high energy multiplication, especially in the case of inertial fusion. (For magnetic fusion, there is also a potential for direct conversion of a-energy into electrical energy.) The $80 \%$ of the $D-T$ power output in energetic neutrons can be captured in a meter-thick external blanket and used for nuclear transmutation, for generation of extremely hot fluids -- or just for maxing steam to drive a conventional power plant. About $2 / 3$ of the D-T fusion neutron output is needed to regenerate the tritium fuel by transmutation of lithium in the blanket. The balance can be used for other forms of nuclear transmutation, such as disposal of long-lived radioactive wastes or 
breeding of fission-reactor fuel. Super-hot fluids could have applications in chemical processing or may be exploitable for direct MHD-generation of electrlcal power. In the present undeveloped state of nuclear fusion technoiogy, the normal practice for conceptual reactor designs studies is to seek economic viability simply in terms of a conventional steam plant. While both magnetic and Inertial fusion reactor plants are most economical in large scale, the standard design goal has been limited to a maximum unit size of $\sim 1000$ MW electric output power.

The outstanding fusion-reactor technology problem is the fabrication and maintenance of the "first wall" through which the energetic neutron flux must pass on the way from the burning plasma to the reactor blanket. Present-day structural materials imply seemingly acceptable -- but inconveniently short -- first-wall replacement times due to radiation damage, resulting in the production of substantial radioactive waste, which fortunately need not be long-lived. Progress in materials research should lead to better performance in both respects. A more radical approach, particularly suitable for inertial confinement, is the use of a first wall made of streaming liquid metal or ceramic pellets. Another possibility is to put the problem back in the area of plasma physics research by looking towards fusion reactions that release most of their energy in charged particles -- while unfortunateiy producing much lower fusion-power densities.

The existence of a wide spectrum of variant solutions constitutes part of the long-term promise of fusion energy. The most direct technological path, however, would seem to be the development of new structural materials that are specialized to the fusion-reactor application. The success of 
magnetic and inertial burning-plasma experiments during the last years of this century may provide the stimulus for an appropriate materials-development program.

Aside from the obvious historical similarities between magnetic and inertial fusion research, there are also some notable differences. One type of asymetry results from the relative age in the two fields. Magnetic fusion reactor concepts were born into an uninhabited ecological niche, where scientific feasibility beckoned as the great objective, and economic feastbility was thought to be an easy corollary. The originally envisaged magnetic fusion reactor schemes -- including the Sakharov-Tamm proposal for the tokamak ${ }^{2}$-. now look so huge and inefflcient that one must marvel that they served to mobilize serious support. During subsequent years, the combination of experimental success and more realistic appreciation of economic factors has caused the size of projected magnetic fusion reactors to decrease substantially. Inertial confinement concepts, on the other hand, were born into a highly competitive world -- already occupied by magnetic confinement concepts. In this case, the conceptual starting point was minimal reactor size, and the experimental learning process has driven a continual growth in reactor-size projections. By these two different evolutionary paths, the magnetic and inertial confinement efforts appear now to have arrived at similarly realistic and promising reactor plans.

A second important difference relates to classification policy. Magnetic fusion research was a closely kept secret until August, 1958, when American-British and Soviet results were unveiled simultaneously in Geneva and found to be roughly identical. During subsequent decades, the international effort in magnetic fusion research has become increasingly 
cooperative and planning is now underway for a joint American-EuropeanJapanese-Soviet project to build an International Thermonuclear Experimental Reactor (ITER). Research in inertial confinement, on the other hand, is still largely classified -- as evidenced by the curiosity that "ind:iectly" illuminated targets are frequently mentioned but seldom described. The relationship between the micro-explosions of inertial confinement and the macro-explosions of nuclear testing has been advantageous in developing physical insight -- while at the same time depriving inertlal confinement researchers of the opportunity to share some of their most significant experimental results.

Recent upward progress in Fig. 1 has been motivated primarily by the fusion application, but there have been a number of important side benefits. First of all, the new field of high-temperature plasma physics has been created, and found to be a scientifically rich and powerful discipline. Laboratory-based plasma physics has provided insight into the Ionosphere and near-space plasma surrounding the earth. The emergence of diagnostic satellites has now turned the earth's neighborhood -- and even the whole solar system -- into a productive large-scale plasma physics laboratory. The advances in plasma physics have also led to unprecedented success in the understanding of astrophysical objects.

Practical applications of high-teraperature plasma physics tend to come In special areas, where the rewards are so great as to justify substantial technological investment. One such area is the extension of lasers into

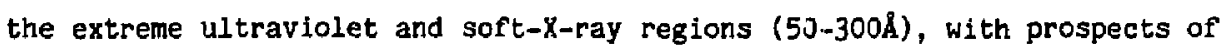
advance to still shorter wavelengths. The techniques of both inertial and magnetic confinement are relevant to this task, and the requirements are sufficiently relaxed compared with those of the fusion energy objective so 
as to promise much earlier economic returns. Important practical applications of X-ray lasers are expected in many fields, including medicine, biology, and semiconductors.

Even in the pre-1950 period, there was one area of research where "hot plasmas," ranging up towards the relativistic plasma regime, were available in the laboratory: namely, the low-density, hot-electron plasmas of high-frequency electronics. Over the years, there has been steady progress towards the generation of shorter wavelength, higher powered microwaves -from magnetrons to gyratrons and recently to free-electron lasers. Some of the same velocity-space Instabilities that interfere with the maintenance of ordered particle motion in magnetic fusion reactor schemes underlie the success of the free-electron laser in extending efficient microwave generation towards the infrared. Among the promising applications of free-electron lasers, in turn, is the heating of magnetic fusion plasmas to ignition by electron-cyclotron absorption of high-frequency microwaves.

The following sections of this paper are designed to provide some technical material in support of the qualitative discussion offered in the introduction. Section 1 reviews the elementary physics concepts of magnetic confinement and describes recent experimental results, with emphasis on tokamak research. Section 2 outlines the basic concepts of inertial confinement and reviews recent (unclassifled) experimental results. Section 3 briefly discusses general applications of high-temperature plasma physics, with emphasis on X-ray lasers. 


\section{Magnetic Confinement}

\section{1 Basic Physical Concepts}

A charged particle gyrating in a magnetic field is equivalent to a diamagnetic dipole with moment $\mu=W_{\perp} / B$, where $W_{\perp}$ is the gyration energy. As illustrated in Fig. 2 for the case of plasma confinement in the earth's magnetosphere, gyrating particles can be "mirror trapped" by positive gradients $\nabla_{\|}$B along magnetic field ilnes, because $\nu$ is an adiabatic invariant, whish is strictly conserved when the gyroradius $r_{B}$ is small compared with the scale helght $L=|B / \nabla B|$. Coulomb scattering, however, can alter $\omega$ and permit the escape of higher-W $W_{\|}$particles along magnetic field lines. From the relative size of the coulomb and fusion cross sections, one sees that unthermalized fusion reactor plasmas -- such as simple mirror-trapped plasmas depending on $W_{1}>W_{\|}--$cannot be expected to produce useful output power, even in the case of $D-T$ fuel at optimal plasma temperature:

One ingenious solution is the tandem mirror machine $e^{3,4}$ where highly energetic ions are mirror-trapped and in turn serve to generate an electrostatic trap for a well-thermalized reacting plasma. A more radical solution is to provide closed magnetic field lines, as in the toroid of Fig. 3, where both mirror-trapped and untrapped particles are constrained to orbit in the neighborhood of magnetic flux surfaces. In that case, the Coulomb scattering rate $\nu_{c}$ leads only to a slow diffusion process, with transport coefficients scaling as $\mathbf{r}_{g}{ }^{2} v_{c}$. This "classical" transport is somewhat enhanced due to orbital excursions larger than $r_{g}$, which occur as the guiding center of the gyromotion ascillates between magnetic mirrors (cf. Fig. 3). In axisymetric toroids, the conservation of canonical angular momentum implies that these excursions must be smaller 
than the gyroradius $r_{g p}$ in the poloidal magnetic field component (the short way around the torus), and classical transport is therefore limited to $D \leq r_{B P}{ }^{2} v_{c}$. The requirement of sufficiently good magnetic confinement to permit the ignition of a D-T plasma then implies a minimum poloidal magnetic flux equal to that of a toroidal current loop carrying several thegamperes. By coincidence, this is also the requirement for confining the large orbital excursions of the $3.5-\mathrm{MeV}$ a-particles that are expected to produne the ignition phenomenon. Classlcal confinement is actually better, by one to two orders of magnitude in the relevant transport coefficients, than the minimum needed for purposes of ignited reactor operation.

A magnetically confined plasma is a diamagnetic medium with a magnetization $n \bar{\mu}$ such that $n \mu B$ equals the plasma pressure $p=n T$. The plasma $\beta$-value $8 \pi n T / B^{2}$ is a measure of plasma diamagnetism -- and therefore also a measure of the plasma's tendency towards Rayleigh-Taylor-like interchange modes, driven by $\mu \nabla_{1} B$. In ideal MHD theory, where the plasma is approximated as a fluid with null electrical resistivity, the resultant locking of magnetic field lines into fluid elements can be used to suppress both the pressure-driven interchange modes and the gross tendency of the plasma current channel towards helical "kinking." Allowance for arbitrarily small but finite resistivity, however, permits field lines to break and rejoin within the fluid, which somewhat aggravates the problem of designing stable configurations with large B-values.

Confined plasmas that are stable against MHD modes can experience slower anomalous loss processes. The nonthermal velocity distributions of mirror-trapped plasmas have a strong tendency towards relaxation by highfrequency modes. Even the isotropic plasmas of toroldal confinement 
necessarily exhibit ordered "drifts" of the particle distribution, corresponding to the diamagnetic magnetization current $\left(J_{m}=c|\nabla(P / B)|\right)$ plus the somewhat slower drift motion of the orbital guiding centers (cf. Fig. 3). These small departures from the Maxwellian can only drive electrostatic microturbulence with a characteristic growth rate that is reduced by the factor $r_{g} / L$ relative to MHD instabilities, but the associated transport can still be as large as the famous Bohm diffusion rate $D_{B o h m}=(1 / 16) \mathrm{cT}_{\mathrm{e}} / \mathrm{eB} \quad \ldots$ which extrapolates to prohibitive reactor-plasma size. A principal goal of toroldal confinement research has been to reduce anomalous transport by several orders of magnitude relative to the Bohin value -- about halfway, on a logarithmic scale, towards the extremely low transport rates of the classical diffusion model.

A number of toroidal confinement configurations ${ }^{5}$ are illustrated in Fig. 4. The "dynamic pinch," which has purely poloidal field and therefore a B-value of unity, is predicted by ideal-MHD theory to be highly unstable. This pessimistic prediction has generally proved to be accurate -- except, perhaps, in the case of recent pinch experiments using very short $\left(10^{-8}-10^{-7}\right.$ sec $)$ current pulses through frozen fibers. 6 The "stabilized pinch" increases the rigidity of the current channel (and lowers the B-yalue into the 10\%-range) by adding a toroida: field componen* $B_{t}$ that is comparable in strength to $B_{p}$. Residual resistive-MHD kink modes serve to maintain a quasi-stable configuration by means of dynamo processes that lower the magnetic energy while conserving the magnetic helicity $\int \mathrm{dV} \overrightarrow{\mathrm{A}} \cdot \overrightarrow{\mathrm{B}}$ : The lowest-energy state is the "reversed-field pinch"7 (RFP), where the toroidai magnetic field outside the plasma is slightly reversed by dynamo currents. The tokamak follows a different strategy of seeking to suppress MHD activity altogether by means of a strong toroidal magnetic 
field $B_{t} \gg B_{p}$. In this case, the toraidal and poloidal periodicity conditions serve to exclude all kink modes, or to localize specific residual modes within limited radial zones. If one wishes to go still further and eliminate the destabilizing effect of the toroidal plasma current entirely, one solution is to use a current-carrying ring, levitated inside the plasma, to generate the confining poloidal field. The "levitron" is well suited for plasma physics research, but not for a D-T reactor. Alternatively, a set of external helical multipole windings can serve to create an average poloidal field. ${ }^{8}$ The "stellarator" concept would lend itself straightforwardly to the desirable objective of a steady-state reactor; by contrast, the tokamak (and RFP) require some auxiliary means to maintain the toroidal clirrent against resistive decay.

\subsection{Progress Towards the Fusion Reactor Regime}

The Lawson diagram of Fig. 5 compares reactor plasma objectives and recent results of magnetic fusion research.9 Economical reactors must operate close to the "burn" curve in Fig. 5, where the ratio $Q$ of fusion power to auxiliary plasma-heating power goes to infinity as the a-particles of the D-T reaction take over the plasma-heating function. The Lawson $Q=1$ curve, refers to breakeven in a fully thermalized plasma with $T_{1}=T_{e}$. When plasmas are heated by injection and thermalization of an energetic non-Maxwellian ion tail, the break-even condition is shown in Fig. 5 to be relaxed appreciably for an ideal ion-energy distribution. (The Lawson diagram for magnetic fusion usually incorporates hydrogenic bremsstrahlung losses -- and therefore has a minimum ignition temperature at $4.4 \mathrm{keV}$.) 
Following the initial years of experimental magnetic fusion research, in the mid-50's, the product $T_{i} \cap T_{E}$ of Table $I$ stood at about $10^{8}$ keV $\mathrm{cm}^{-3} \mathrm{sec}$. By the early 60 's, it had risen to $10^{10} \mathrm{keV} \mathrm{cm}^{-3} \mathrm{sec}$. In 1969 , the T-3 tokamak, at the I.V. Kurchatov Institute, advanced the $T_{i} \cap \tau_{E^{-v a l u e ~}}$ to $5 \cdot 10^{11} \mathrm{keV} \mathrm{cm}^{-3} \mathrm{sec}$ in a device of modest parameters (major radius $R=150 \mathrm{~cm}$, plasma current $I_{p} \sim 100 \mathrm{kA}$ ). During subsequent years, worldwide progress along the tokamak line has continued steadily (cf. Fig. 5).

The key to the advance of tokamak data points in the Lawson diagram has been the improvement of the quality of toroidal confinement. The rigid magnetic field structure of the tokamak eliminated MHD-activity as a dominant driver of anomalous transport. At the same time, the simplicity of the tokamak magnet configuration, and the effectiveness of its built-in ohmic-heating mechanism permitted the production of much larger and denser plasmas in the T-3 device than had been available in contemporary levitron and stellarator experiments. The tokamak edge region, where $\nabla$ (log p) becomes large, and where atomic processes are an important part of the energy and particle balances, has electrostatic fluctuation levels (e $\delta \phi / T$, $\delta n / n)$ that are comparable to those of pre-tokamak plasmas and are accompanied by a Bohmlike level of anomalous transport. The novelty of the tokamak plasma consists in the emergence of a relatively quiescent hot-plasma core, where local transport is sufficiently small so that the resultant global energy confinement time $\tau_{E}$ exceeds the Bohm confinement time by orders of magnitude and comes close to classical expectation.

A dramatic demonstration of this favorable trend was provided by the Alcator tokamak experiments, carried out at MIT, beginning in the mid-70's. In tokamak devices somewhat smaller than $\mathrm{T}-3$, the product of 
plasma density times linear size $I$ was raised by using strong magnetic fields ( $(10 \mathrm{~T})$ and correspondingly intense ohmic heating. These experiments yielded $T_{i} \cap \tau_{E}$-values in the range $10^{13}-10^{14} \mathrm{keV} \mathrm{cm}^{-3}$ sec. They also led to the empirical "neo-Alcator" scaling law where $\tau_{E}$ does not increase simply in proportion to $\mathrm{L}^{2}$, as in ordinary classical diffusion processes, but increases additionally with the product $\ln$ (up to a maximim value that is limited by other loss mechanisms). This optmistic cubic size-dependence was subsequently verified in the much larger TFTR experiment at Princeton and in the JET experiment at Culham, England (cf. Table II and Fig. 6). JET has measured record ${ }^{\top} \mathrm{E}^{\text {-values, }}$ close to one second, and TFTR has reached an $n r_{E^{-v a l u e}}$ of $1.5 \cdot 10^{14} \mathrm{~cm}^{-3}$ sec (cf. Fig. 5 ).

The inherent ohmic-heating process of the tokamak raises the plasma temperature into the $1-5 \mathrm{keV}$ range, but for purposes of bringing the plasma to the ignition point, the introduction of auxiliary heating appears to be convenient, Tokamak plasmas have been heated (with roughly comparable effectiveness) by the injection of energetic neutral-atom beams and by the absorption of various electromagnetic waves -- particularly in the frequency ranges around the ion and electron gyrofrequencies. Auxiliary-heating experiments have proved enlightening in respect to the scaling of anomalous transport: In ohmic heating, the input power $\mathrm{P}_{H}$ can be increased only by raising the plasma current, and therefore also the confining poloidal field. In auxiliary heating, the input power can be raised for fixed magnetic confinement parameters, and the "Goldston" scaling of ${ }^{\top} \mathrm{E}$ was found: An adverse $\mathrm{P}_{\mathrm{H}}^{-1 / 2}$-dependence, together with favorable dependences on size and poloidal field strength $B_{p}$. When the $\mathrm{P}_{\mathrm{H}}$-dependence is eliminated (using $\mathrm{P}_{\mathrm{H}} \propto \mathrm{L}^{3} \mathrm{nT} / \tau_{E}$ ), one recognizes that the 
Goldston scaling fits into the family of Bohmlike scaling laws, but with a much lower magnitude of transport and with the familiar B-dependence of $D_{\text {Bohm }}$ replaced by a $B_{p}$-dependence.

The Goldston scaling would be compatible with an ignited tokamak plasma in the 30-MA range of plasma current -- which is not unrealistic for a full-scale commercial reactor -- but would impose a serious budgetary handicap during the phase of research and development. This problem could be resolved by means of relatively small numerical improvement factors in the Goldston transport coefficient $(-2)$, which happily appear to be available through plasma-edge-control techniques.

The ASDEX experiment in Garching, Germany used a magnetic divertor (Fig. 7) to inhibit the reinjection and reionization of outflowing plasma, and found that at sufficiently high heating-power levels the plasma regime became thermally bistable. Starting from the auxillary-heated ohmic regime, which they named the L-mode, the plasma was able to "Jump" Into the $H$-mode, which also exhibits a Goldston-type scaling for ${ }^{t}{ }_{E}$, but with several times longer confinement times. These divertor H-mode experiments have been extended successfully to the 10-MW level of neutral-beam-heating power in the DIII-D tokamak at GA Technologies in San Diego, and also in the JET device, yielding the "JET" data point shown in Fig. 5. While the TFTR is not equipped with a divertor, edge-plasma recycling was effectively suppressed by using a large surface of absorbent graphite tiles as the plasma limiter. In this way $\tau_{E}$ was also improved severalfold, giving the high-T 1 TETR point in Fig. 5 (which has somewhat lower electron temperature $\mathrm{T}_{\mathrm{e}}-7 \mathrm{keV}$ ). 
The improved TFTR regime has given D-D neutron yields of $10^{16} \mathrm{sec}^{-1}$, with pulse durations of order $1 \mathrm{sec}$, which would correspond to a total fusion energy release of about $4 \mathrm{MJ}$ if $\mathrm{D}-\mathrm{T}$ fuel sare gubstituts for D-D. The energy-multiplication factor $Q$ would then fall into the range $0.20-0.25$. A number of machine improvements are currently underway on TFTR, as well as on JET and on the JT-60 device in Ibaraki-ken, Japan, which seem likely to permit the achievement of "equivalent" $0 \sim 1$ conditions in D-D plasmas during 1988. Break-even experiments in D-T plasmas are expected to begin in TFTR during 1990 and in JET during 1992.

Beyond the present generation of experimental tokamaks, international planning is looking towards a major tokamak test reactor facility to produce quasi-steady-state ignited-plasma conditions and to provide an initial test bed for fusion reactor technology. A foint international design for such a machine (the INTOR ${ }^{10}$ ) was developed during the period 1979-87. Extended experience with cooperative international design and supporting research efforts is now providing the basis for the initiation of a three-year design program (1987-90) aimed at the joint construction of the ITER (International Thermonuclear Experimental Reactor) device during the period 1993-2000. At present, there are also a number of parallel national design projects, aimed at similar or somewhat smaller reactor facilities.

The ITER class of tokamak designs is characterized by superconducting steady-state magnet systems that produce $\sim 5-T$ magnetic fields at the plasma. (Superconducting-coil operation is to be prototyped during the late $80^{\prime} \mathrm{s}$ by the T-15 tokamak at the Kurchatov Institute, and the Tore Supra at Cadarache, France.) Other characteristic ITER parameters are: major radius $\mathrm{A}$ - $5 \mathrm{~m}$ and plasma current $\mathrm{I}_{\mathrm{p}}-15 \mathrm{MA}$. 
The success of the Alcator experiment in achieving relatively high $T_{i} n \tau_{E}$ in small machine size has opened up a parallel path to ignition, which is expected to prove valuable for exploring the physics of burning plasmas preparatory to operating a large engineering test reactor like the ITER. By raiging the field strength $B$ to $10 \mathrm{~T}$, one can expect to achieve essentially the same ignition margin as the ITER (with very nearly the same set of dimensionless plasma physics parameters) at about half the plasma current. The US fusion program has therefore adopted the Compact Ignition Tokanak ( $B=10 \mathrm{~T}, I_{p}=9 \mathrm{MA}, R=1.75 \mathrm{~m}$ ) as its principal near-term contribution to the international tokamak reactor effort. The Ignitor device, being studied as part of the Italian fusion program, is designed to provide similarly strong magnetic confinement parameters (B $-13 \mathrm{~T}$, $I_{p}$ $\left.10 \mathrm{MA}\right)$ in still smaller size $(R-1.0 \mathrm{~m})$, but to be less directly prototypical of an engineering test reactor.

Looking beyond the ITER towards the comsercially oriented phase of tokamak reactor development, the quality of plasma confinement becomes a less critical issue than the fusion power density, which depends on the plasma beta value. Theoretical understanding in this area of MHD stability analysis is well advanced and has led to fruitful geometric ideas -- for example, the D-shaping of the tokamak minor plasma cross section, as in Figs. 6 and 7 . Experimental tokamaks of this geometry have already reached average B-values exceeding $6 \%$ (cf., Fig. 8), wich is ample for ITER and CIT, but would be on the law side for a 5-T tokamak poker reactor. The most exciting theoretical idea in the area of B-maximization is entry into the "second stability regime," where rising plasma pressure deforms the equilibrium magnetic field configuration in such a favorable way that MHD stability actually improves. Initial experiments in this direction (using "bean-shaped" tokamak cross sections) are currently underway. 
A second important direction of advance is tawards steady-state reactor operation. A tokamak current is readily induced by pulsed transformer coils, but the finite resistance of the plasma toroid, together with the finiteness of the transformer volt-seconds, implies a limited pulse duration. Tokamak plasmas in present-day devices typically last 1-10 seconds. For plasmas of reactor size and temperature, "guasi-static" tokamak pulses of several hours duration could be reached by the transformer technique. To achieve a true steady-state, non-inductive current drive, for example by tangentially injected neutral beams or phased microwave grids, appears to be readily possible. A 2-MA current has been driven in the JT-60 device by means of $3 \mathrm{MW}$ of 2-GHz microwaves. Because of the previously noted tendency of non-Maxwellian velocity distributions to relax collisionally on the same time scale on which fusion power is produced, the introduction of auxiliary power for steady-state current drive unfortunately tends to lower the effective Q-value of tokamak reactors. In this context, a very favorable prediction of classical tokamak transport theory is that, at sufficiently high values of "beta poioidal" ( $B_{p}=8 \pi n T / B_{p}{ }^{2}$ ) the diffusive expansion of the plasma itself will drive most of the plasma current. This "boatstrap effect" has begun to be seen experimentally in TFTR regimes with $B_{p}=2-3$.

In sum, the prospects for advancing the tokamak concept towards ideal reactor performance are substantial and promising -- but they are not yet so solidly founded as to discourage serious competition from other sectors of magnetic confinement research. In regard to the achievement of high 6-values, the RFP version of the stabilized pinch (Fig. 4) can easily outdistance present-day tokamak performarice -- maintaining gross stability at the 10-20\% level. Multi-MA RFP experiments, aimed at extending plasma 
confinement parameters into the "tokamak range" of the Lawson diagram (Fig. 5), are currently under construction at Padua, Italy and Los Alamos, New Mexico.

The stellarator (Fig. 4) offers the unique advantage of true steady-state confinement without need for any auxiliary power input. The quality of confinement in stellarators has been found similar to that in tokamaks -- though the greater complexity of stellarators has caused them to lag behind tokamaks in affordable plasma size, and therefore also to lag behind in the Lawson diagram. Currently, new stellarators with 200-kA-level equivalent poloidal confining fields are entering operation in Oak Ridge, Tennessee, and Garching, Germany, and a substantially larger stellarator facility is being planned for Nagoya, Japan.

A tandem-mirror reactor could have the special attraction of combining steady-state operation with high b-value. Fairly complex magnetic-field architecture and auxiliary power systems are required to provide sufficiently good confinement for net power production, but theoretically the mirror reactor concept remains a viable contender. During the past several years, funding of the U.S. mirror program has declined sharply, but research on the mirror confinement of hot plasmas continues actively in Novosibirsk, USSR, and Tsukuba, Japan -- as well as in the magnetospheres of Earth and other planets.

\section{Inertial Fusion}

\subsection{Basic Physical Concepts}

Since the generation of fusion power depends on the product $n_{E}$ (cf., Fig. 5), the energy conflnement time $\tau_{E}$ can be allowed to become very short, provided that the density $n$ is sufficlently high. An 
inertially confined (i.e., dynamically expanding) fuel pellet of mass density $\rho$ and radius $R$, burning at a prescribed temperature, has an $n \tau_{E^{-s c a l i n g}}$ that is proportional to $\rho R$ (since $n \propto \rho$ and $\tau_{E} \propto R$ ). The investment of thermal energy is proportional to the total fuel mass of the pellet $M \propto \rho R^{3}=(\rho R)^{3} \rho^{-2}$. The energy needed to meet a given $n \tau_{E^{-}}$ requirement is seen to decrease as $\rho^{-2}$ with rising density.

In quantitative terms, an approximate equivalent of the Lawson $D-T$ ignition condition at $4 \mathrm{keV}$ is $p R_{c} \sim 0.3 \mathrm{gm} \mathrm{cm}^{-2}$, where $\mathrm{R}_{c}$ is the radius of the hot core of the pellet. In order that ignition may actually take place, most of the a-particle energy should be deposited within the pellet core, thus imposing an auxiliary minimum-size condition that depends on $p R_{c}$ and again happens to be satisfied for $\rho R_{c}-0.3 \mathrm{gm} \mathrm{cm}^{-2}$ (the a-particle range in 10-keV $\mathrm{D}-\mathrm{T}$ ).

The favorable o-scaling of the energy investment required to reach ignition and high gain suggests a strategy of compressing most of the pellet mass isentropically to the maximum possible density. ${ }^{11-14}$ To minimize the specific energy investment required for compression, the fuel temperature must be kept near the Fermi energy, which scales as $E_{E} \approx n^{1 / 3}$ (cf. Fig. 1). The "cold" bulk of the pellet can be ignited by careful shaping of the driver pulse, so as to create a small, hot central fuel core.

The proposed experimental approach to the achievement of ignition is illustrated in Fig. 9. A high-powered, short-pulse driver is used to heat the pellet surface, thus forming an expanding atmosphere. As this spherical "rocket blow-off" proceeds, the remainder of the pellet is compressed by the recoil. At maximum compression, the pellet core crosses 
the $\rho R_{c}$-threshold for ignition. The temperature climbs rapidly into the 10-keV range during an a-particle-driven thermal excursion that spreads outward through the rest of the pellet.

The fusion energy multiplication requirements that are relevant to the case of inertial confinement can be displayed in a Lawson-type diagram (Fig. 10) that resembles the usual Lawson diagran of Fig. 5. The parameter $\tau_{E}$ now refers to the duration of the compressed plasma state, rather than to the time constant of heat replacement by non-radiative transport in a steady-state plasma. The ignition or burn curve, which is the locus of $Q=\infty$ operation in the steady-state case, is now the threshold condition for energy multiplication. The ignition curve itself is somewhat more lenient in the inertial case: Thermal bremsstrahlung can be trapped in dense plasmas, so that the minimum ignition temperature is below the 4.4-keV threshold for the optically thin case treated in Fig. 5. Defining an inertial "D-T pellet gain" $G_{D T}$ as the fusion energy release divided by the energy investment in the ions and electrons of the target material, one has $G_{D T}-(3 \mathrm{MeV} / T) \phi$, where $\phi$ is the burn-up fraction, which is approximately proportional to $n \tau_{E}$ or $p R$, when is small. The $D-T$ gain indicated in Fig. 10 for a plasma core that lgnites with $o R_{c} \sim 0.3,0 \mathrm{~cm}^{-2}$ and heats up into the $10-\mathrm{keV}$ range is of order 10-20, corresponding to a burn-Lp fraction of 3-6\%. If the $o R$ of the pellet as a whole is larger by an order of magnitude than that of the ignited core, the final burn-up fraction increases and the gain is also improved, because the surrounding fuel is initially colder. The reactor goal indicated in Fig. 10 is $G_{D T} \sim 10^{3}$. It is useful to define the "target gain" $G_{T}=\pi G_{D T}$ (the fusion yield divided by the total energy input from the driver system) in terms of the coupling efficiency $\eta$, which includes the hydrodynamic rocket 
efficiency and the fractional absorption of driver energy at the pellet surface. The magnitude of $n$ is of order $10^{-1}$ for the reactor illustration, so that $G_{T}$ is of order $10^{2}$-- leaving some room for the internal losses in the driver system and the overall power plant.

A pellet structure that lends itself well to the ignition and burn process is a thin spherical plastic shell of outer radius $R_{0}$, containing $a$ layer $\Delta R$ of iiquid $D-T$ (for example, in the form of a liquid-saturated low-2 sponge), inside of which there is a spherical cavity of radius $R_{0}-\Delta R$, filled with equilibrlum $D-T$ vapor, During the pelletcompression phase (which typically occurs on a $10^{-8}$-sec time scale with an associated terminal velocity of $3-4 \cdot 10^{7} \mathrm{~cm} / \mathrm{sec}$ ) the average density of the liquid shell rises at least $10^{3}$ fold (from $0.2 \mathrm{gm}^{-3}$ to $-200 \mathrm{gm}^{-3}$ ), while its temperature remains at several times the Fermi energy, reaching a final value somewhat below 1 keV. The low-density central cavity collapses, producing a 5-keV plasma core or "spark plug" that ignites (on a $10^{-11}$ sec time scale) and sends a burn-wave traveling outward through the colder bulk of the pellet. Pressure balance causes the density to be somewhat reduced within the hottest region. The core radius required to satisfy the ignition condition is of order $R_{c} \sim 30-100 \mu \mathrm{m}$, the mass of the "spark plug" is of order $10^{-5}-10^{-4} \mathrm{gm}$, and the assaciated thermal energy investment is $10-100 \mathrm{~kJ}$. To achieve the desired targetenergy multiplication by virtue of a propagating burn, the radius of the surrounding pellet material should be of order $\rho \mathrm{R} \geqslant 3 \mathrm{gm}^{-2}$, with a mass of several times $10^{-3} \mathrm{gm}$, corresponding to an additional energy Investment of several hundred kilojoules. For such target parameters, the fuel burn-up fraction $\$$ reaches $30-40 \%$. 
The successful formation of a small, hor, central ignition region (with $R_{\mathrm{e}} / \mathrm{R}_{\mathrm{o}}$ typically a few times $10^{-2}$ ) is perhaps the most critical physics challenge of inertial confinement research. Initial asymetries associated with either the pellet or the illumination tend to be amplified exponentially by a Rayleigh-Taylor-type instability mechanism. When a light fluid $\rho_{1}$ pushes a hoavy one $\rho_{h}$ to generate an acceleration $\pm g$, interchange modes are unstable with a growth rate $(\mathrm{Akg})^{1 / 2}$, where $k$ is the wave number and $A$ is the Atwood number $\left(p_{h}-p_{1}\right) /\left(\rho_{h}+p_{1}\right)$. This type of unstable situation arises both during the inttial acceleration process and during the final "zolcration, shore the nonvergir: l.plle pellet material compresses the small central cavity of hot plasma. Asymmetrles that have exponentiated during the initial phase can lead to turbulent mixing of hot and cold fuel during the final phase, thus preventing 1gnition. The most dangerous instability wave numbers $k$ are of order $\Delta R^{-1}$, where $\Delta \mathrm{R}$ is the (strongly reduced) shell thicleness following initial acceleration. The associated number of $\bar{c}$-foldings during compression is proportional to the square root of the "in-flight aspect ratio," i.e., $(\mathrm{R} / \mathrm{LR})^{1 / 2}$. The use of high-aspect-ratio shells is constrained by a maximur permissible number of about elght e-foldings, set by the limitations of target-fabrication technology. Mitlgating factors result from the finiteness of the density gradient and from ihe nature of the rocket blow-off mechanism itself: As the ablation front eats into the pellet material, it tends also to carry away the vorticity associated with Rayleigh-Taylor interchanges. If the velocity characterizing the ablation front is comparable to $(\mathrm{g} / \mathrm{k})^{1 / 2}$, a significant stabilizing effect can result. In this context, careful shaping of the driver-power pulse as a function of time is found to be advantageous. 
The mativation for the use of pellets with large $R / \Delta R$ arises from the severity of the requirements on the driver system. The energetic efficiency of the pellet compression would be highest if the implosion velocity were to exceed the rocket blow-off velocity somewhat. The pellet, being denser than the surrounding plasma atmosphere, tends, however, to implode more slowly -- resulting in a hydrodynamic "rocket efficiency" that can easily fall below $10 \%$, unless $R / \Delta R$ is large. This effect becomes more severe with rising laser intensity, thus giving an additional advantage to pellets that are more hollow, with relatively larger initial radius $R_{0}$ and lower surface-power density requirements. Aside from the aspect ratio $\mathrm{R}_{\mathrm{o}} / \Delta \mathrm{R}$, the depth of penetration of the pellet illumination has an important effect on the compression process: Absorption of the driver energy in a deeper, denser layer of the plasma corona surrounding the pellet tends to blow off more material at lower rocket velocity -- two features that are valuable, respectively, for inhibiting Rayleigh-Taylor modes and improving hydrodynamic efficiency.

For an illustrative laser-driver energy output of $10^{6} \mathrm{~J}$, delivered in $10^{-8}$ sec onto a pellet of radius $R_{0}=3 \mathrm{~mm}$, the required power density would be of order $10^{14} \mathrm{~W} \mathrm{~cm}^{-2}$. As the laser light prapagates into the edge of the pellet, it encounters a local plasma frequency $\omega_{p}$ that increases proportional to $n^{1 / 2}$-- until propagation cuts off at the critical density $n_{c}$ where $\omega_{p}$ equals the light frequency. (For $1-m i c r o n ~ l i g h t, n_{c}$ is about $10^{21} \mathrm{~cm}^{-3}$, so that cutoff occurs very near the pellet surface.) The "classical" process for absorbing the laser light is inverse bremsstrahlung, where the input energy is thermalized collisionally. This process is favored by using shorter laser wavelengths, which give deeper penetration and allow the energy to be deposited into denser, colder pellet 
material. On the other hand, in the hot, lower-density plasma corona surrounding the pellet, illumination at very high power densities lends itself to the excitation of a variety of plasma waves (cf. Fig. 11), which have largely undesirable effects on the pellet-compression process. The most serious consequences are: (1) back-scattering of incident light, which reduces the absorption efficiency; (2) channeling of light into local density-depressions, which spoils the illumination symatry and aggravates phenomena associated with high laser intensity; and (3) plasma-wave acceleration of energetic electrons, which causes premature propagation of heat into the bulk-pellet material, and interferes with isentropic compression. The streaming of non-uniformly produced energetic electrons is one of several mechanisms that can give rise to the generation of very strong magnetic fields $\left(B-10^{2} \mathrm{~T}\right)--$ which are generally unhelpful in the ICF context, since reduced surface heat conductivity teids to interfere with efficient, uniform absorption of the driver energy.

A promising alternative to direct laser-pellet illumination is to heat up the interior surface of a hohlraum, with the resultant radiation "bath" acting to illuminate and compress an interior pellet. Since the thermal radiation in the hohlraum is in the soft $X$-ray range, the energy deposition onto the pellet surface is relatively penetrating, which improves the rocket efficiency. The primary process of laser-heating at the hohiraum surface will, of course, tend to excite the usual plasma instabilities, but this problem is mitigated by the use of surface materials with high atomic number, which enhances the collisionality of the plasma. Indirect illumination also lends itself conveniently to various alternative drivers, such as energetic ion beams: a hohlraum has the obvious potential for 
improving the uniformity of pellet illumination. The drawback of "indirect drive" is that it adds one more energy-conversion stage, thus tending to lower the ultimate efficiency of the coupling process.

\subsection{Progress Towards the Fusior Reactor Regine}

The somewhat idealized theoretical concepts of inertial confinement that were current during the late 1960's envisaged compressed pellet densities of order $2 \cdot 10^{3} \mathrm{gm} \mathrm{cm}^{-3}$-- some $10^{4}$ times ordinary íquid density -- and estimated that, for peak powers of order $10^{14} \mathrm{~W}$, driver energies of order $10 \mathrm{~kJ}$ (or even less) might suffice to reach ignition. Delivering an energy pulse of this magnitude on a sub-nanosecond time scale seemed to be within the reach of the emerging solid-state laser technology. By the mid-70's, neodymium glass laser facilities in the 10-100 J, $10^{19}-10^{12} \mathrm{~W}$ range became available and provided the initial body of experimental information on laser-pellet interactions at a wavelength of 1 ำ.

As in the case of magnetic fusion research, the earliest experiments were oriented towards fusion-neutron production. Thin-walled D-T-gas-filled glass "microballoons" were imploded, and measurable neutron yields were obtained. These experiments departed fundamentally from the theoretical strategy for reaching ignition (cf. Sec. 3.1), in that high temperatures were produced at relatively low densities, by exploding the outer glass shell and generating a convergent spherical shock wave to heat the central D-T fuel. While these "exploding pisher" experiments were not on the direct path to energy production and did not address the critical physics issues of ablation and fluid stability, they have provided valuable experience with laser-irradiation physics and diagnostics for the study of the compression process. 
By the end of the $70^{\prime} \mathrm{s}$, neutron yields in the range $10^{10}$ per shot were being produced in the SHIVA experiments at the Lawrence Livermore National Laboratory (LLNL), with oriver energies in the range 1-10 $\mathrm{kJ}$. By 1985, the OMEGA laser (cf. Table III) at the University of Rochester, New York, reached over $10^{11}$ neutrons per pulse, the GEKO program at Osaka, Japan then reached $10^{12}$, and both GEKO XII and the NOVA facility at Livermore have meanwhile exceeded $10^{13}$. In the latest experiments, ${ }^{9}$ central temperatures

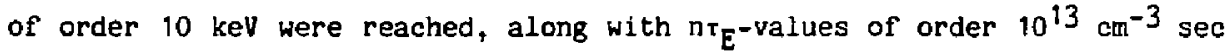
(cf., the high-temperature points in Fig. 10). The associated target gains, however, were only of order $10^{-3}$, and it would seem difficult to reach the break-even range (equivalent to $Q=1$ for magnetic fusion) by further development along this line.

Alongside the microballoon experiments, the reactor-relevant compression technique described in Sec. 3.1 has been pursued with increasing realism and success. Laboratory experiments to date have avoided the complexities of actualiy using liquid-D-T-filled pellets, but have otherwise addressed the physics issues involved in the ablative compression of Rayleigh-Taylor-unstable shells. Densification factors as high as 130 (relative to liquid $D-T$ ) have been achieved in the NOVA facility, by using indirect drive for the ablative compression of thick gas-filled shells. Experiments of this type have provided the high-ntE points in Fig. 10. The corresponding peak plasma p: essures (Fig. 13) are of order $-3 \cdot 10^{10}$ atmospheres - far beyond all other laboratory experience, and well on the way towards the inertial reactor goal of $10^{11}-10^{12}$ atmospheres. 
These NOVA experiments have successfully addressed another figure of merit that is of fundamental importance for achieving ignition: the convergence ratio $R_{o} / R_{c}$, relating the initial pellet radius to the final radius of the hot pellet core. Computer-modeling studies have shown that for purposes of substantial energy multiplication, the convergence ratio should be greater than 40 . In recent NovA experiments, convergence factors exceeding 35 (i.e., a fuel volume reduction of $4 \cdot 10^{4}$ ) have been reported. The somewhat unexpected achievement of this result, with less-than-optimal driver-pulse shaping, would seem to support the concept of Rayleigh-Taylor-mode inhibition and has favorable implications for high-gain experiments. The indirect-drive results represent a major improvement relative to those reported for direct-drive experiments in NOVA, where convergence ratios greater than 10 were accompanied by a marked deterioration of actual vs, calculated neutron yield -- indicative of unstable mixing and cooling in the hot core.

Hohlraums (like tokamaks) have opened a major path to experimental success -- but in inertial (as in magnetic) fusion research, there is still ample room for alternative approaches to claim the potential for ultimate economic advantage. In particular, there is a persuasive case that the direct laser-illumination approach would lend itself to high-gain operation with reactor systems that are less demanding in regard to driver efficiency and energy output. 9

The development of laser drivers was initially hampered by a defect of experimental implementation: the excitation of excessive plasma turbulence at the illuminated surface. With 1-micron neodymium-laser light, the coupling to various plasma waves was found to be so strong, relative to the classical inverse-bremsstrahlung process, that the absorption efficiency 
dropped to the 30\% level for the desired illumination intensities in the $10^{14}-10^{15}-\mathrm{Wcm}^{-2}$ range. Thanks to improvements in the frequencymultiplication technique at the University of Rochester and at the École Polytechnique in France, the neodymium light was converted efficiently to its 3 rd and 4 th harmonics, respectively, with an attendant rise of lasertarget absorption into the range above 70\%. Most important, there was a marked accompanying drop in the fraction of laser energy delivered to the undesirable energetic-electron tail. The outstanding results achieved in the GEKO and NOVA experiments were obtained with the frequency-multiplied neodymium-glass laser light (at $1 / 2$ and $1 / 3$ microns) -- which was found to be advantageous for both direct and indirect drive. By contrast, the $\mathrm{CO}_{2}$-laser approach uses a driver system that would be highly attractive from the point of view of reactor engineering, but unfortunately lases at 10 microns. The $\mathrm{CO}_{2}$-laser experiments encountered such severe plasma-interaction problems with both direct and indirect drive that this particlar alternative (formerly pursued in the ANTARES program at Los Alamos and the LEKO program at Osaka) has now been discontinued.

A more fundamental challenge to the use of direct laser illumination is the requirement for a high degree of uniformity. During the past several years, a number of promising "optical-smoothing" schemes have been proposed to counteract imperfections of the laser-beam source. The basic strategy is to split the laser light into a large set of randomly phased independent "beamlets," each of which produces nearly perfectly uniform illumination over a small area. Thousands of such beamlets could be focussed onto the pellet surface, the interference between neighbors could be kept small, and the desired quality of overall illumination could theoretically be achieved. These Ideas are being tested in the PHAROS III laser facility at 
the Naval Research Laboratory in Washington, D.C., the GEKO Iaser at Osaka, and the OMEGA laser at Rochester, as well as at the Shanghai Institute of Optics in China. In the NRL approach, ranoiom time delays have been introduced among beamlets, with a view to combining spatial and temporal incoherence so as to avoid a stationary interference pattern.

The reactor concept envisaged for the direct-laser-drive approach is compatible with a moderately efflcient short-wavelength source, such as the krypton-fluoride laser system (source efficiency $\sim 7 \%$, wavelength $1 / 4$ micron), which is currently under development at Los Alamos and elsewhere. The iraprovement in target gain that is expected for the direct-drive approach would give this reactor system an overall energy multiplication comparable to that achieved by the indirect approach when using a 20\%-efficient source, such as an energetic ion-beam driver (or possibly a 10\%-efficient source, with a compensating improvement in X-ray energy-conversion efficiency.) In either case, one envisages a reactor with about 1-GW electric output power. For the currently expected minimum driver-input energies into the pellet, which are in the 3-10 $\mathrm{MJ}$ range, the fusion miaroexplosions would have yields of order 0,3-1 GJ and corresponding repetition rates of $3-10 \mathrm{~Hz}$.

If the direction of future inertial-confinement research is towards indirect ion-beam drivers, there are two main possibilities. Light ions (such as lithium) have been accelerated into the-low MeV range in the PBFA experiments at the Sandia National Laboratory in New Mexico. The PBFA II facility is designed to yield 1-2 MJ of 20-30 MeV ions at a peak power of $10^{13}-10^{14} \mathrm{~W}$. Another promising alternative is to use 10-GeV-range ions of somewhat greater mass $(A \sim 200)$, with the high nuclear charge helping to 
reduce their stopping distance. Appropriate accelerator technology is being developed at the Lawrence Berkeley National Laboratary, but no largescale prototype driver has as yet been undertaken.

\section{General Applications}

The rapid progress in high-temperature plasma physics that has taken place thanks to the stimulus of the world's long-term energy problem has created major new scientific and technological resources for other areas of practical application. One important lllustration is the development of X-ray lasers -- which has drawn on the expertise of both inertial and magnetic fusion research.

For such purposes as X-ray microscopy, holography, and surface etching, lasers capable of operating at wavelengths of - $40 \AA$ (energies of $~ 300 \mathrm{eV}$ ) would be of great value, and even the soft $X$-ray range around 100-200 $\AA$ has interesting applications. The plasma-temperature requirements are much less demanding than for fusion: Depending on the nature of the population-inversion scheme, electron temperatures $T_{e}$ in the low keV range lend themselves to the requirements for a $40-\AA$ laser, and temperatures as small as a few hundred eV can suffice for the longer-wavelength range. (The ion temperatures $T_{1}$ are desired to be somewhat lower than $T_{e}$, so as to avoid excessive Doppler broadening, which reduces the gain of the medium.)

The optimal plasma densities for the X-ray laser application are intermediate between those of magnetic and inertial fusion: typically, $\mathrm{n}=10^{19}-10^{22} \mathrm{~cm}^{-3}$, with the higher densities corresponding to shorter $X$-ray wavelengths. The plasma density is limited on the high side by the need to avoid collisional depopulation of excited energy levels, but the 
choice of $\mathrm{n}$ is driven upward -- especially in the case of short wavelengths $\lambda$-- by the desire to maximize the gain $G$ (which is proportional to $n \lambda^{a}$, where $2 \leq a \leq 4)$.

The burdensome condition on the energy confinement time $t_{E}$ that preoccupies fusion researchers has no precise counterpart in the $X$-ray laser application. If the $X$-ray laser scheme is based on the "electron-pump" mechanism, 15 where population inversion is achieved by collistonal filling of upper energy levels and radiative depletion of lower ones, there is no evident advantage in prolonging the heat-up time $\tau_{H}$. When a laser scheme based on three-body ion-recombination is used, 16 the best strategy is to cool the plasma electrons as rapidly as possible, following the initial heating and ionization phase, while seeking to keep the plasma density from falling (i.e., requiring the ratio of energy to particle confinement time, $\tau^{\prime} \tau^{\prime}{ }^{\prime}$ to be as small as possible). A scaling that is at least somewhat reminiscent of Lawson's $n^{\tau} E^{-c o n d i t i o n ~ c a n, ~}$ however, be elicited by requiring the gain length GL to be sufficiently large (of order 20), so as to saturate the lasing medium. Defining $T_{L}=L / c$ as the transit time of the laser pulse over the path length $L$ through the medium, the GL-requirement translates into an $n \tau_{L}$ " requirement. This type of nt-criterion could make sense, for example, when the gain length is limited by the number of passes that can be made between a pair of xuv-mirrors before the amplifying medium is lost; even for X-ray laser parameters as large as $\mathrm{nL}=10^{23} \mathrm{~cm}^{-2}$, however, the $\mathrm{n \tau}_{\mathrm{L}}$-requirement amounts to a relatively lenient $3 \cdot 10^{12} \mathrm{~cm}^{-3} \mathrm{sec}$. (For single-pass amplification, there is no requirement that the lifetime of the plasma should exceed $\tau_{L}$, since the driver-power input can be timed to move along $L$, keeping in phase with the $X$-ray pulse.) 
The X-ray laser experiments carried out at LLNL ${ }^{15}$ using the NOVETTE laser driver in 1984, and more recently, using two of the ten NOVA beams, have led the way toward the achievement of high gain lengths. As illustrated in Fig. 14, the targets consist of thin foils $(-0.1 \mu \mathrm{m})$. The heated foil material provides very uniform transient $\left(<10^{-9} \mathrm{sec}\right)$ plasmas up to $5 \mathrm{~cm}$ in length. Initial success came by using plasmas of $T_{e} \leqq 1 \mathrm{keV}, \mathrm{n} \sim 5 \cdot 10^{20} \mathrm{~cm}^{-3}$ for electron-pumping of neon-like selenium, thus producing gain lengths of order 8 at wavelengths of $206 \AA$ and $209 \AA$. The peak gain length of these lines has now been raised to 15-20, corresponding to a remarkable $10^{6}$-fold amplification relative to the spontaneous emission level. Three other lines with smaller gains have also been observed -- including the $182-\AA$ line which had inftially been expected to dominate. Other neon-like systems have been used to produce wavelengths down to $106 \AA$, and nickel-like systems have gone down to $50 \AA--$ but the gain lengths in these short-wavelength cases are in the range $\leq 4$.

A quite different approach to $\mathrm{X}$-ray lasers, inspired by the magnetic confinement experience, has been followed at PPPL ${ }^{16}$ (cf. Fig. 15). The basic idea has been to strip light ions, such as carbon, and use the recombination mechanism to produce laser amplification with relatively predictable hydrogen-like systems. In inertially confined plasmas, where the time scale ${ }^{T}$ for the desired drop in electron temperature is the same as the time scale $\tau_{p}$ for the undesired drop in plasma density, this approach tends to be very inefficient, but the introduction of a strong longitudinal magnetic field to confine the plasma column helps make $\tau_{p}$ much longer than the time required to cool the electrons by radiation from "impurity ions." In the PPPL experiments, a 3-GW, 300-J, $\mathrm{CO}_{2}$-laser is used to produce carbon plasmas of $200-300$ ev initial electron temperature and 
flelds stronger than those within the target ions themselves and may be able to induce short-wavelength lasing action in systems excited by multiphoton processes.

In the K-ray laser application, the plasma serves only to provide a suitable environment for an excited ion population that lases according to its own spectroscoplc rules. During recent years, a number of other interesting techniques have been invented where plasma particles are drectly responsible for exciting (or damping) coherent electromagnetic radiation -- usually with much greater efficiency than can be achieved with a laser system that must work through the intermediary agency of an excited ion population. These collective wave-particle interactions are of a type long studied in magnetic fusion schemes that depend on strongly ordered motion -- for example, mirror machines.

The free-electron laser 17,18 (FEL) imparts a transverse wiggle to a high-quality energetic electron beam, by means of a periodic magnet structure. The FEL electron population is unneutralized, but when seen in its rest frame, looks just like an ordinary plasma. The periodically accelerated electrons radiate collectively, transferring their kinetic energy into coherent electromagnetic waves. With a magnet system that is "tapered" so as to keep in phase with the slowing-down electron beam, efficiencies as high as 30-40\% have been achieved. Both short-pulse and steady-state FEL's have been demonstrated. The emitted frequencies can range up from the microwave domain into the visible (or higher), depending on the energy of the wiggled electron beam.

Among the many promising applications of the FEL is the high-powered heating of magnetic fusion plasmas at the electron gyrofrequency (or its harmonics). Another possibility is to invert the FEL scheme in the form of 
an electron-beam accelerator (driven by FEL's). Still another ingenious concept uses the interference of two input laser beams to excite high-electric-field heat waves at the plasma frequency of an ordinary neutralized plasma, thus providing a powerful electron acceleration structure. The particular inventions mentioned here are part of a larger continuum of new ideas that holds the promise of producing a plasma technological revolution during the coming decades.

\section{Acknowledgments}

I am indebted to the inertial confinement research community -. particularly to Drs. S.E. Bodner, E.M. Campbell, D.A. Goerz, and R.L. MeCrory -- for their help in preparing this paper. Work supported by U.S. Department of Energy Contract No. DE-ACO2-76CH03073. 


\section{References}

1 "Physies Through the 1990's. Plasmas and Fluids" (National Academy Press, Washington, D.C., 1986).

2 I.E. Tamm and A.D. Sakharov in "Plasma Physics and the Problem of Controlled Thermonuclear Reactions" (M.A. Leontovich, ed.) I, 1, (Pergamon, Oxford, 1961).

3 G.I. Dimov et al., Sov. J. Plasma Phys. ?. 326 (1976).

4 T.K. Fowler and B.G. Logan, Comments Plasma Phys. Controlled Fusion Res. $\underline{2}, 167$ (1977).

5 Fusion, (E. Teller, ed.) Part A, I (Academic Press, New York, 1981).

6 J.D. Sethian et al., Phys. Rev. Lett. $\underline{59}, 892$ (1987).

7 J.B. Taylor, Phys. Rev. Lett. 33, 1139 (1974).

8 L. Spitzer, Jr., Phys. Fluids 1, 253 (1958).

9 Plasma Physics and Controlled Nuclear Fusion Research, (Proc. 11th International Conference, Kyoto, Japan, 1986) 3 Volumes, IAEA, Vienna, 1987.

10 International Tokamak Reactor, Phase Two A, Part II, (International Atomic Energy Agency, Vienna, 1986).

11 J. Nuckolls, Phys. Today, 35, 24 (1982).

12 R.L. MeCrory and J.M. Soures, Selentific American 225, 68-79 (August 1986).

13 N.G. Basov et al., Sov. Phys. JETP $\underline{19}, 123$ (1964).

14 J. Nuckolls et al., Nature 239, 139 (1972).

15 D.L. Matthews et al., Phys. Rev. Lett. 54, 110 (1985).

16 S. Suckewer et el., Phys. Rev. Lett. 55, 1753 (1985).

17 D.A.G. Deacon et al., Phys. Bev. Lett. 38, 892 (1977).

18 T.J. Orzechowsk: et al., Phys. Rev. Lett. 57, 2172 (1986). 
TABLE I.

Comparison of $T_{i}{ }^{n} E^{-V a l u e s}$ (in keV $\mathrm{cm}^{-3} \mathrm{sec}$ )

\begin{tabular}{lcc}
\hline & $\begin{array}{c}\text { Magnetic } \\
\text { Confinement }\end{array}$ & $\begin{array}{c}\text { Inertial } \\
\text { Confinement }\end{array}$ \\
\hline Best Experiments & $3 \cdot 10^{14}$ & $3 \cdot 10^{14}$ \\
Break-even & $5 \cdot 10^{14}$ & $5 \cdot 10^{15}$ \\
Ignition & $5 \cdot 10^{15}$ & $-10^{16}$ \\
Target Gain $=1$ & & $-10^{17}$ \\
Target Gain $=100$ & & \\
\hline
\end{tabular}

* Reached by self-heating, following ignition. 
TABLE II.

Some Major Tokamak Facilities

\begin{tabular}{llccll}
\hline Facility & Location & $\begin{array}{c}\text { Major } \\
\text { Radius } \\
(\mathrm{m})\end{array}$ & $\begin{array}{c}\text { Field } \\
\text { Strength } \\
(\mathrm{T})\end{array}$ & $\begin{array}{c}\text { Plasma } \\
\text { Current } \\
(\mathrm{MA})\end{array}$ & $\begin{array}{l}\text { Special } \\
\text { Feature }\end{array}$ \\
\hline ASDEX-U & FRG & 1.6 & 3.9 & $(2.0)^{*}$ & Divertor \\
DIII-D & US & 1.7 & 2.2 & $2.5(5)^{* *}$ & High B \\
Tore Supra & France & 2.2 & 4.5 & $(1.7)^{*}$ & Superconducting \\
T-15 & USSR & 2.4 & 4.5 & 2.0 & Superconducting \\
TFTR & US & 2.5 & 5.2 & $2.5(3)^{* *}$ & D-T \\
JT-60 & Japan & 3.0 & 4.5 & 2.7 & Divertor \\
JET & U.K. & 3.0 & 3.5 & $5.0(7)^{* *}$ & D-T \\
& & & & & \\
\hline
\end{tabular}

* Not yet in experimental operation

**Potential for extended operation 
Some Major Inertial Confinement Facilities

\begin{tabular}{lllcc}
\hline Facility & Location & $\begin{array}{l}\text { Driver } \\
\text { Type }\end{array}$ & $\begin{array}{c}\text { Energy } \\
\text { (kJ) }\end{array}$ & $\begin{array}{c}\text { Power } \\
\text { (TW) }\end{array}$ \\
\hline VULCAN & UR & Nd-glass** & 1 & 2 \\
PHAROS II & IJS & Nd-glass** & 1.5 & 1 \\
OMEGA & US & Nd-glass** & 4 & 12 \\
DELPHIN & USSR & Nd-glass** & 2 & 1 \\
GEKKO XII & Japan & Nd-glass** & 20 & 40 \\
PHEBUS & Erance & Nd-glass** & 24 & 20 \\
NOVA & US & Nd-glass** & 120 & 100 \\
AURORA & US & KrF & $(5)^{*}$ & $(1)^{*}$ \\
PBFA-II & US & Light ion & $(2000) *$ & $(100)^{*}$
\end{tabular}

* Not yet in experimental operation

* Output parameters are for $1 \mu \mathrm{m}$ and should be roughly halved for 2nd and 3rd harmonics. 


\section{Figure Captions}

1. Comprehensive density-temperature diagram for plasma physics. Two illustrative experimental data points recently obtained in magnetic and inertial confinement research have been added.

2. A charged particle can be trapped in the earth's dipole magnetic field, if its velocity is predominantly perpendicular to $\bar{B}$. The particle gyro-orbit precesses around the equator.

3. In a toroidally symmetric magnetic field configuration, particles of all velocity vectors can be confined. Particles trapped in the weaker-field region on the large-R side of the torus precess in the toroidal direction.

4. A number of toroidal confinement configurations have been studied experimentally. The dynamic pinch has a purely poloidal field, generated by a toroidal plasma current. Otner configurations use the combination of a toroidal magnetic field plus a poloidal field component generated by a toroidal current flowing in a solid ring coil (levitron) or within the plasma itself (tokamak and stabilized pinch). The stellarator uses external helical multipole windings to generate an average poloidal fiesd somponent. 
5. The Lawson diagram presents the requirements for equilibrium burn $(Q=\infty)$ and for breakeven $(Q=1)$ in thermalized plasma. Non-Maxwelliam plasmas with enlarged energetic-ion components can, in principle, achieve breakeven under substantially related conditions. The "TETR $Q=1$ objective" illustrates an experimental break-even goal.

6. The vacuum chamber of the JET device has approximately the crosssectional size that will be required for a tokamak reactor -- but the major radius will have to be approximately doubled.

7. Tokamak confinement properties can be improved by D-shaping the minor cross section of the magnetic surfaces. The associated magnetic separatrix can be used to divert the plasma outflow into the "scrape-off layer."

8. The tokamak beta-value (ratio of plasma pressure to magnetic pressure $\left.\mathrm{B}^{2} / 8 \pi\right)$ is limited by $\mathrm{MHD}$ stability conditions that can be relaxed by cross-sectional shaping. (The approximate 8 -values that can theoretically be achieved with various shapes are indicated.) Present experimental points and probable reactor requirements are shown.

9. In the inertial confinement approach, high-powered laser beams vaporize the surface of a pellet, which is compressed by the recoil, reaching high central temperatures and densities. The compressed D-T plasma ignites and burns. 
10. An inertial confinement diagram analogous to Lawson's shows a similar ignition condition. The practical reactor objective of 100-fold target gain calls for substantial advance beyond the ignition curve.

11. Intense illumination by laser light can give rise to a variety of collective plasma interactions.

12. The 4.4-m diameter target chamber of the NOVA facility is designed for ten-beam laser-light input.

13. Plasma pressures in the $10^{11}-10^{12}$-atmosphere range will be needed for an inertial confinement reactor. The $10^{10}$-atmosphere range has already been reached by the experiments.

14. By means of a cylindrical lens, the NOVA laser beam can be used to produce a thin column of hot plasma from a metal-foil target. For suitable choice of ion species and plasma parameters, a longitudinal $X$-ray laser bean is generated within this plasma column.

15. A strong magnetic fleld can be used to confine a thin colum of hot plasma evolving from a laser-1lluminated solid target. A tubular X-ray laser beam is generated at the radiation-cooled surface of the plasma column. 


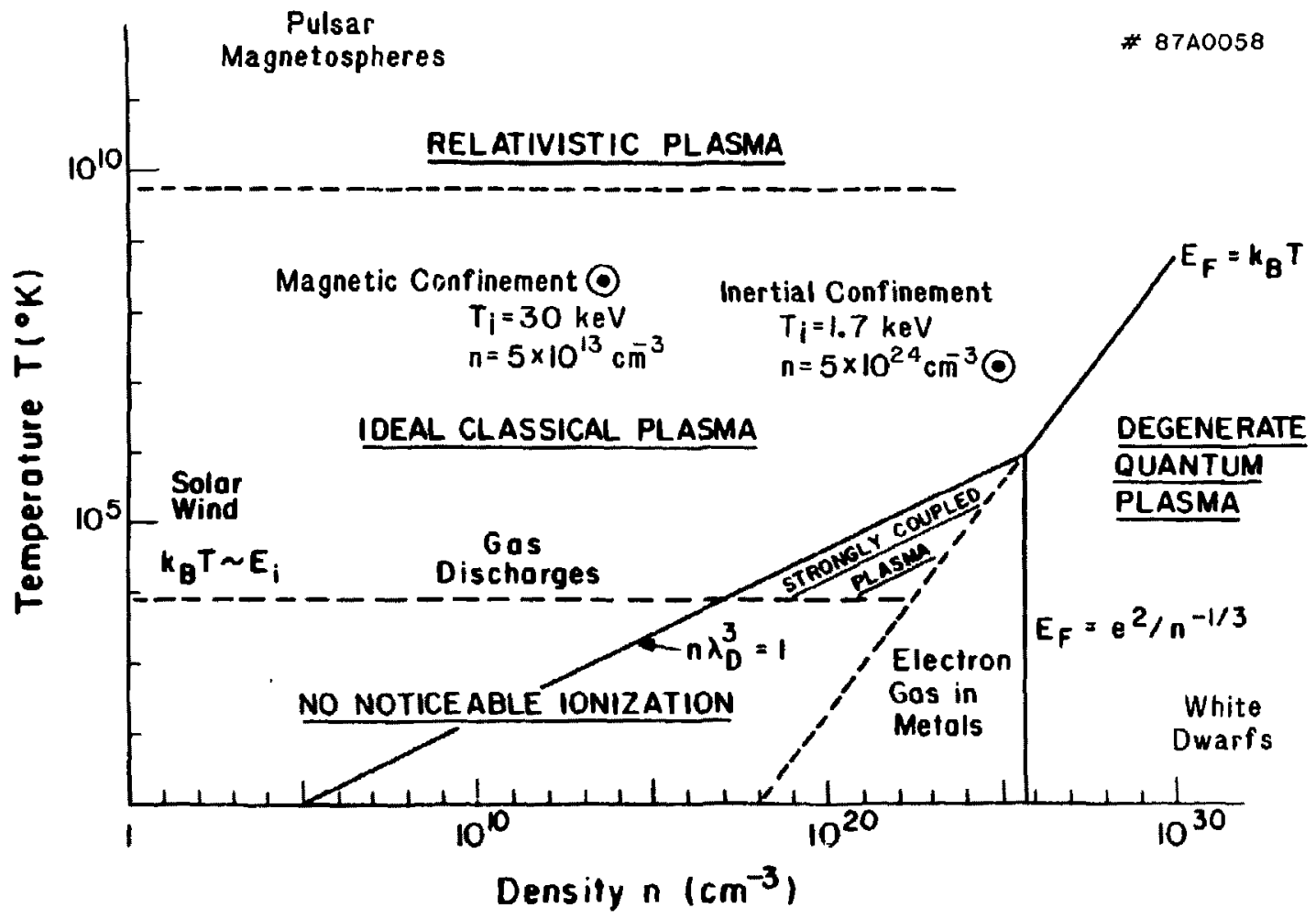




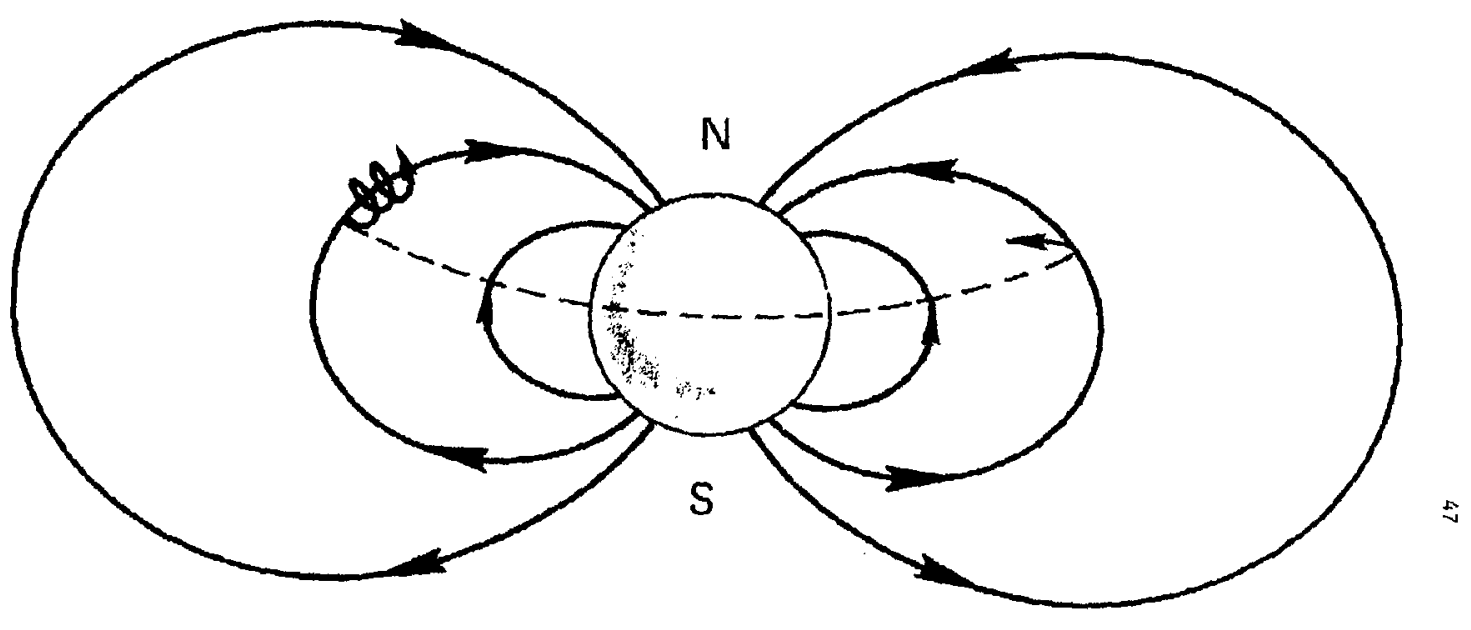

FIg. 2 


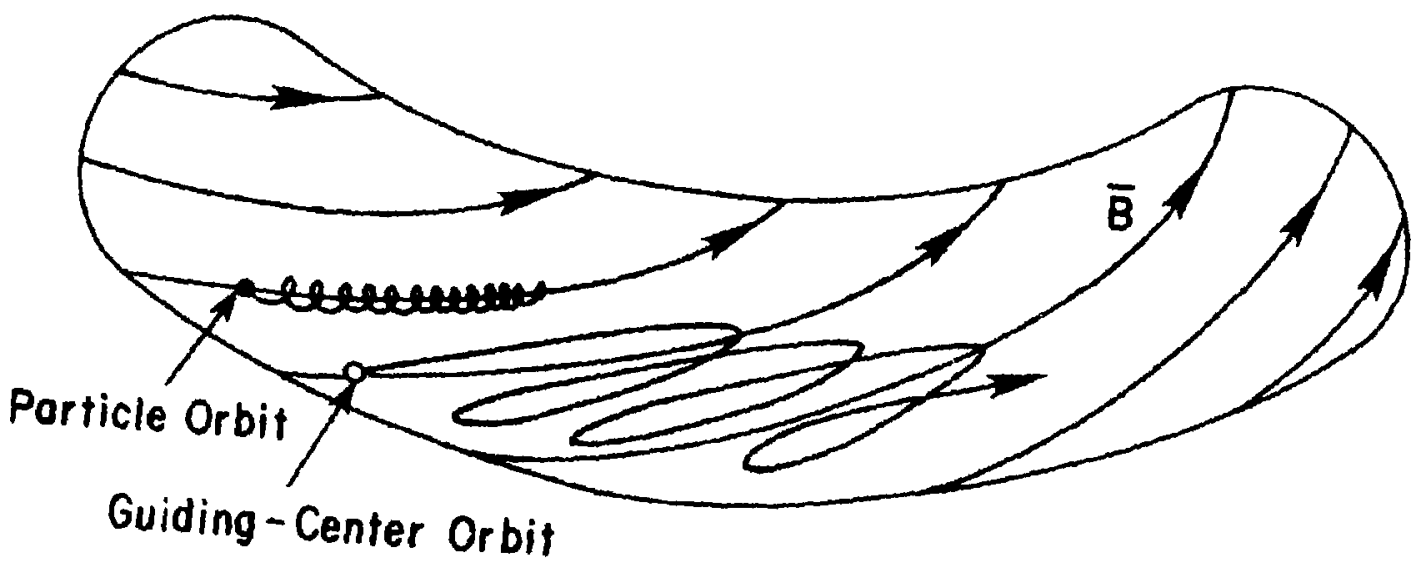


\#81 $\times 0378$

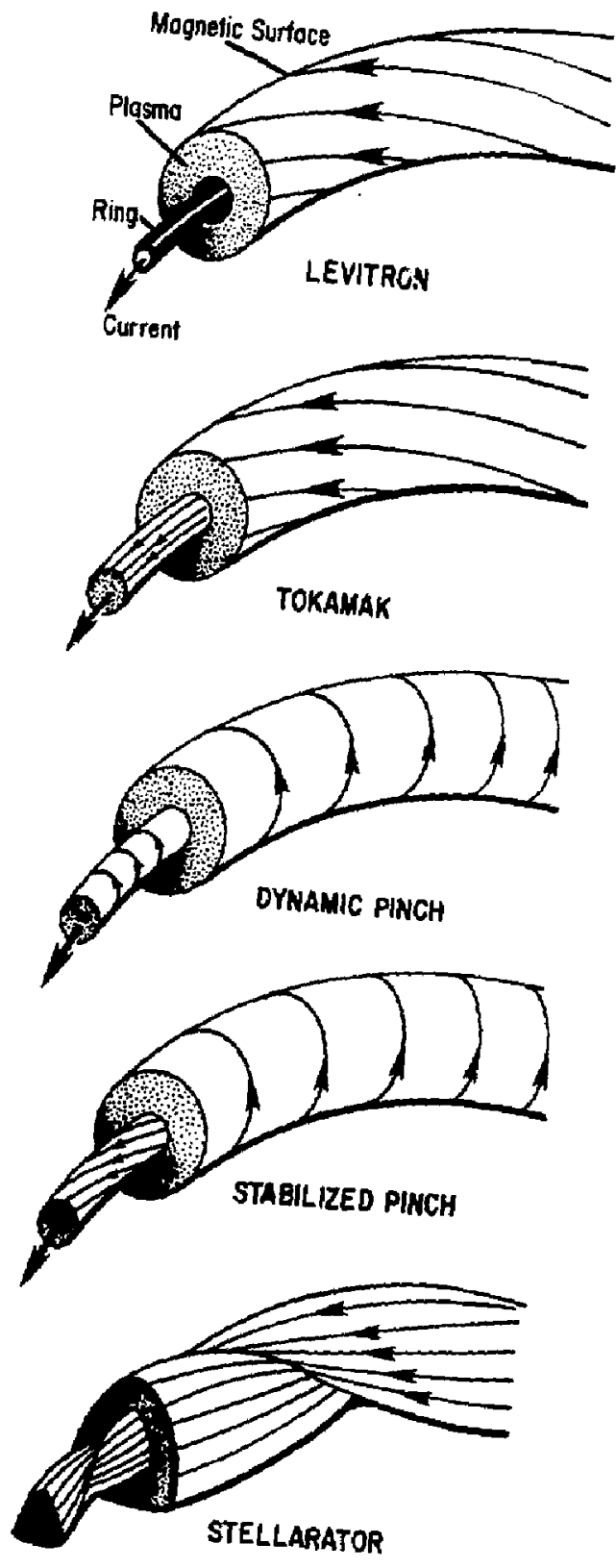

FIg. 4 


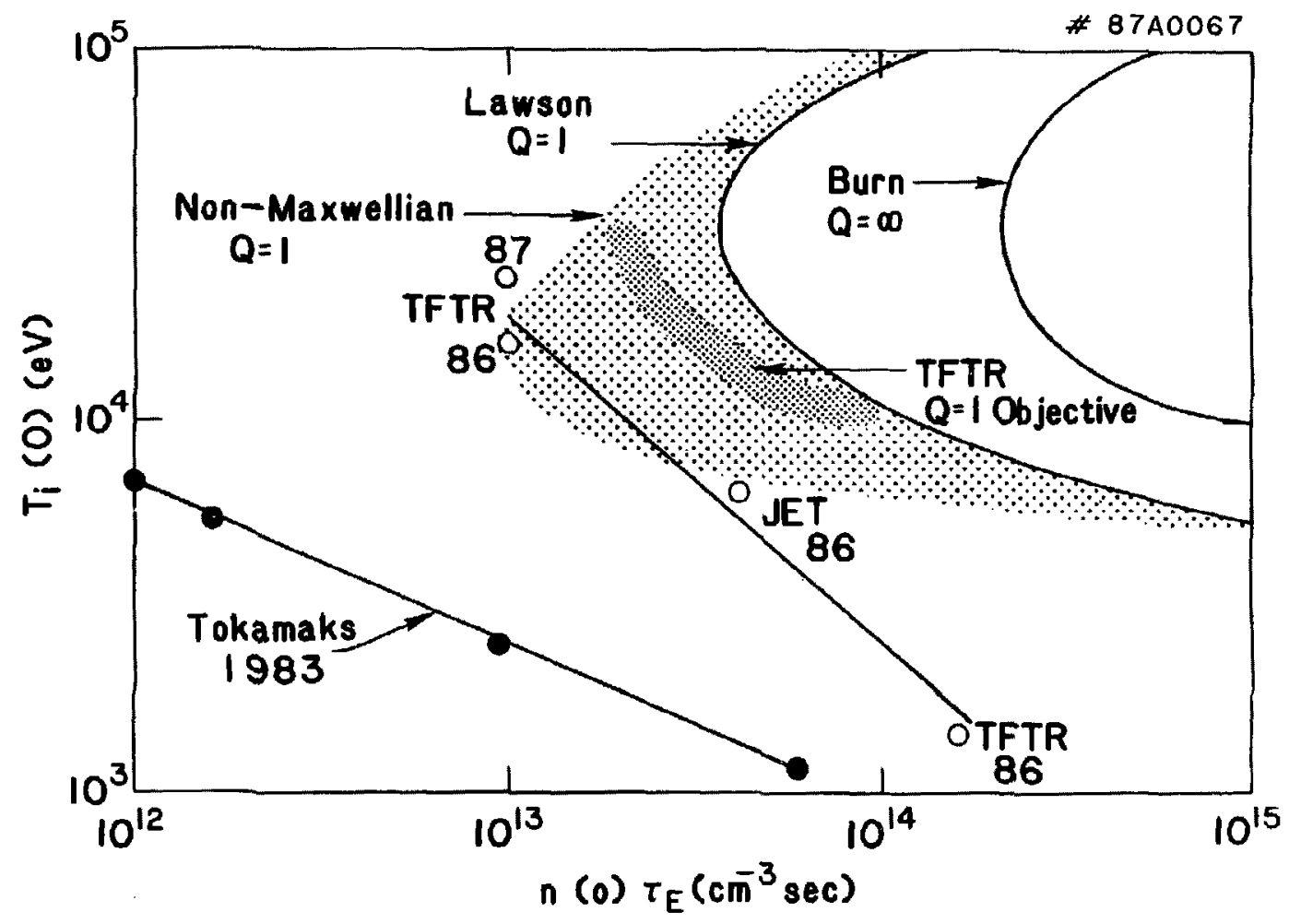

Fig. 5 


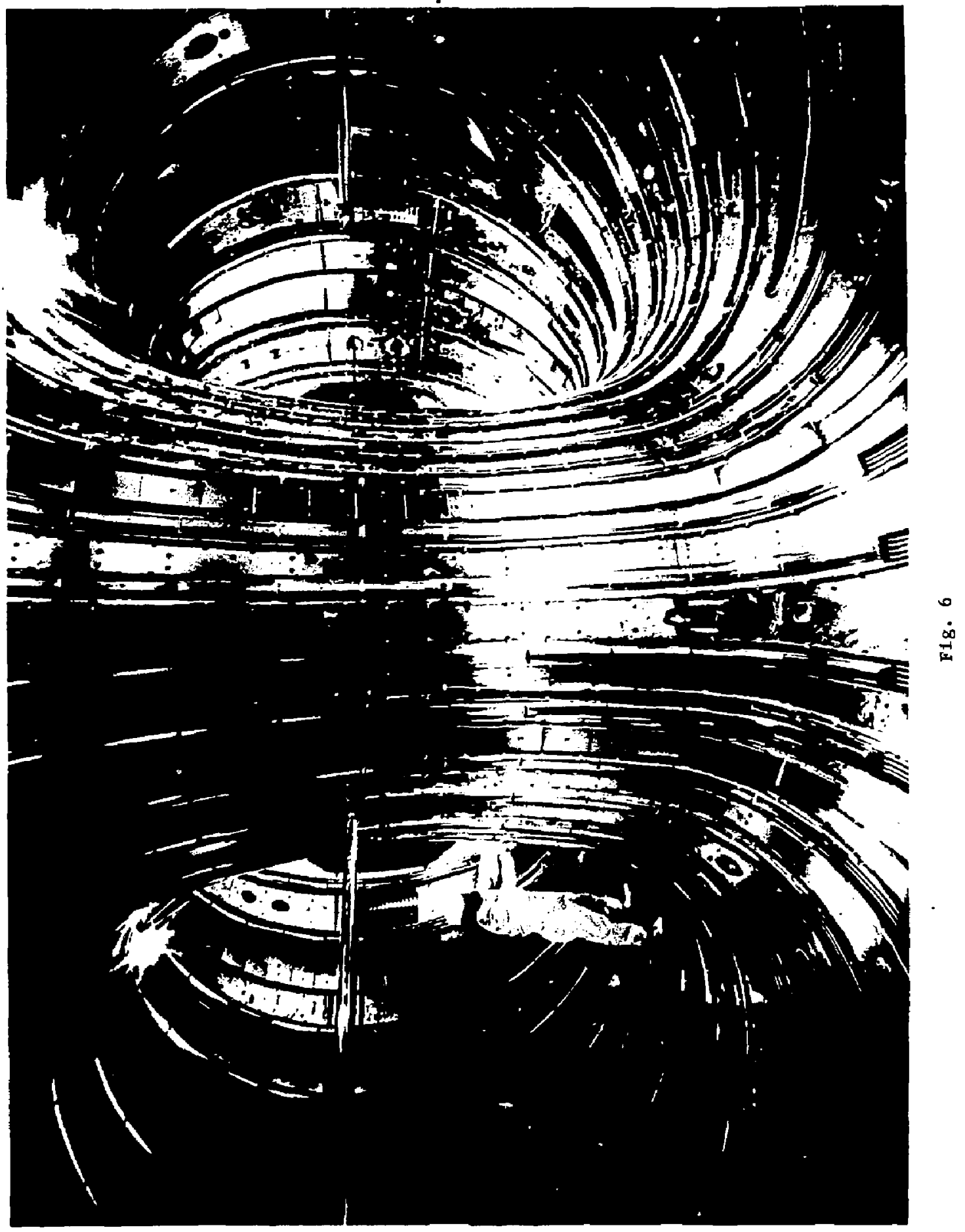


\# 87P0118

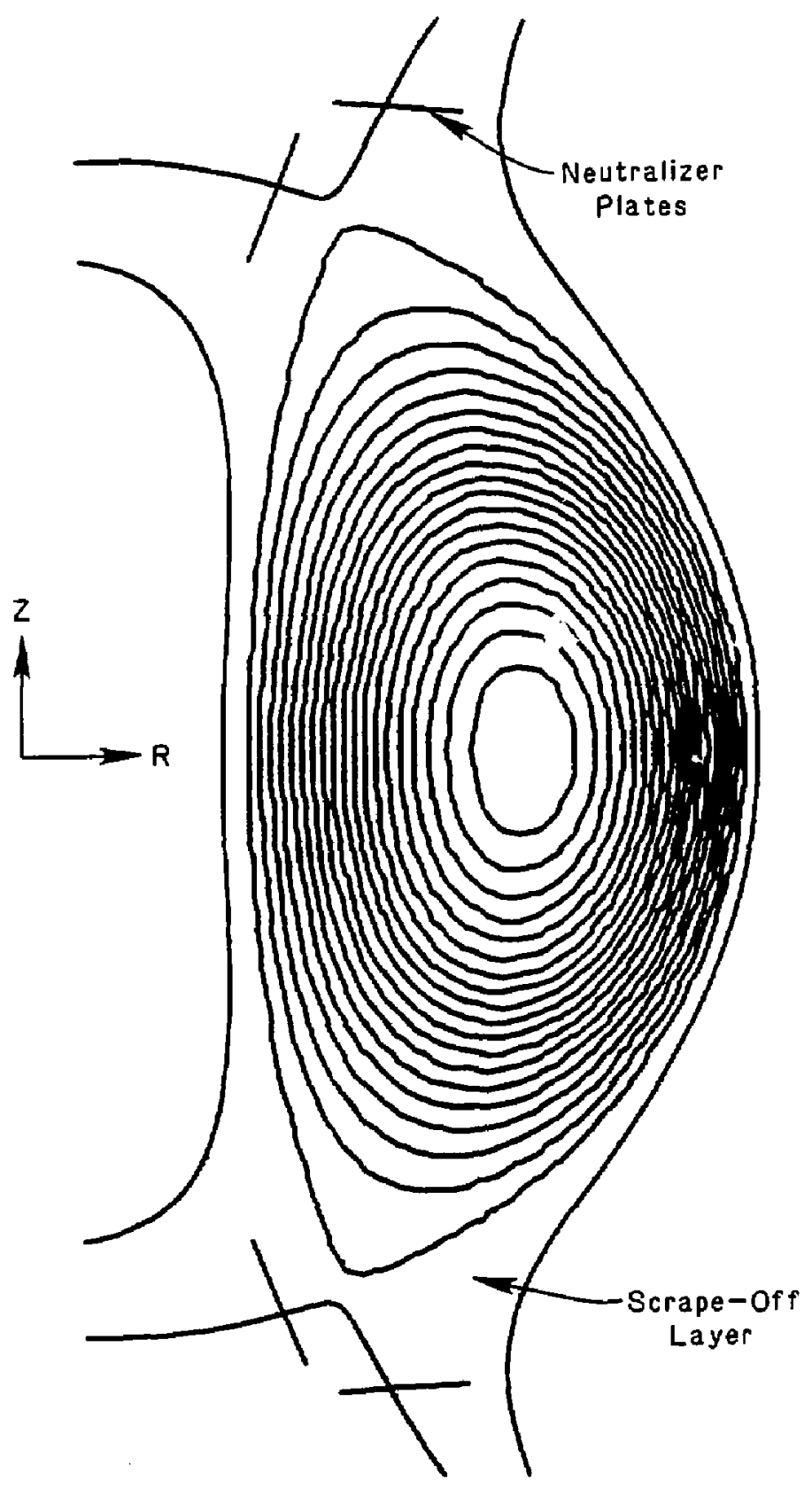

F1g. 7 


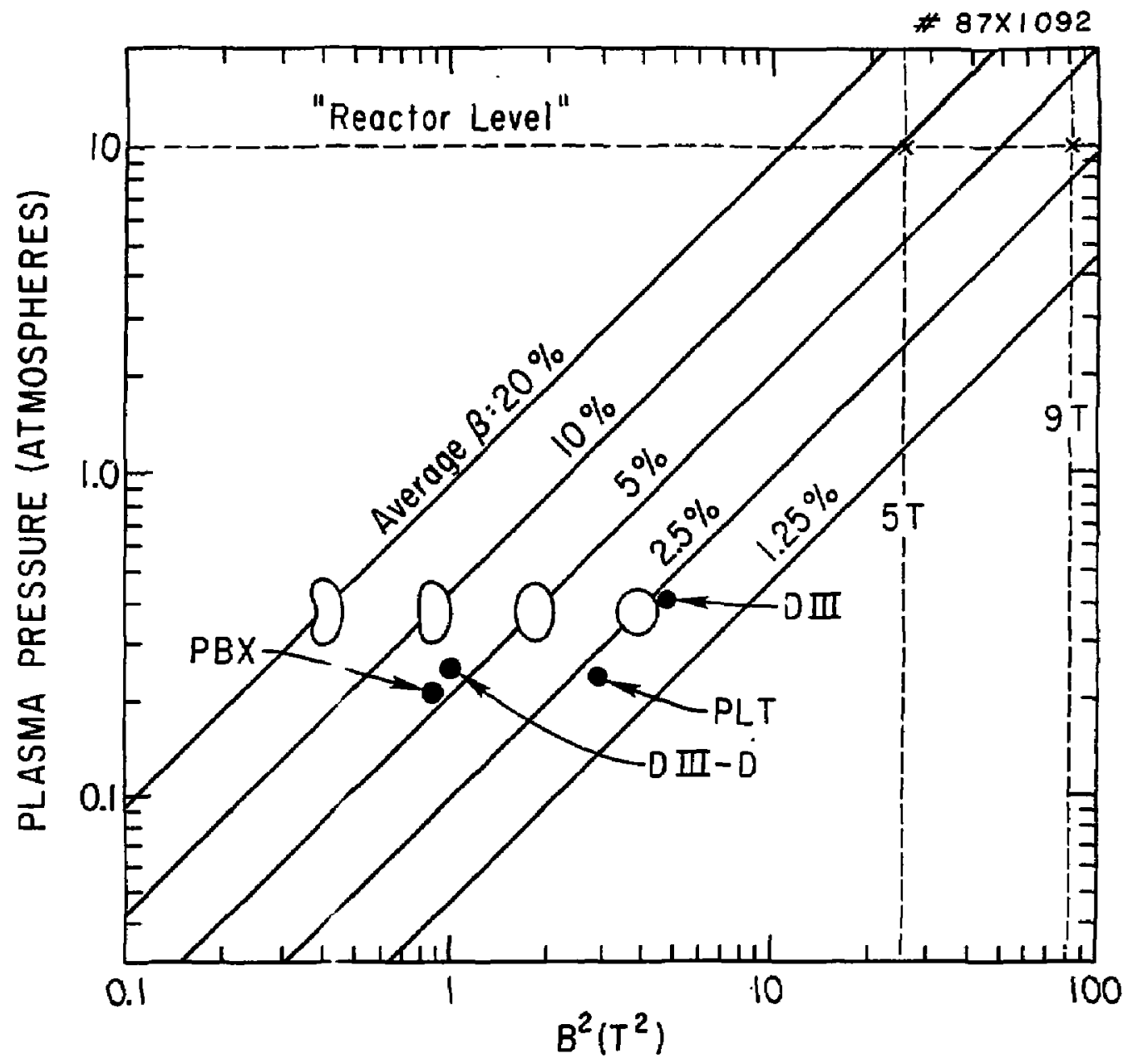

Fig. 8 


\section{Luer energy \\ Inward transported Hun thermal enorgy}

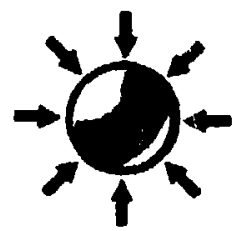

Atmosphere Formation

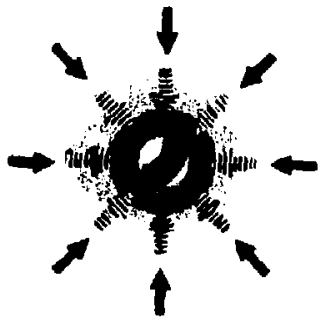

Compression

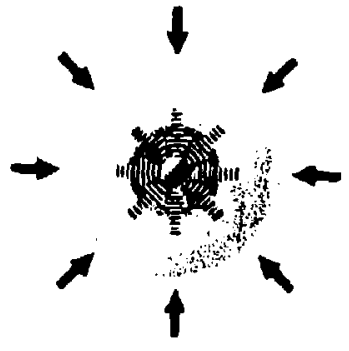

Ignition

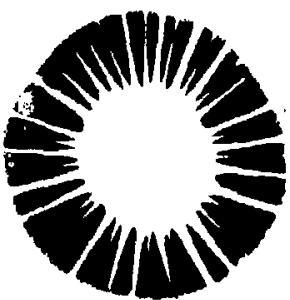

Burn 


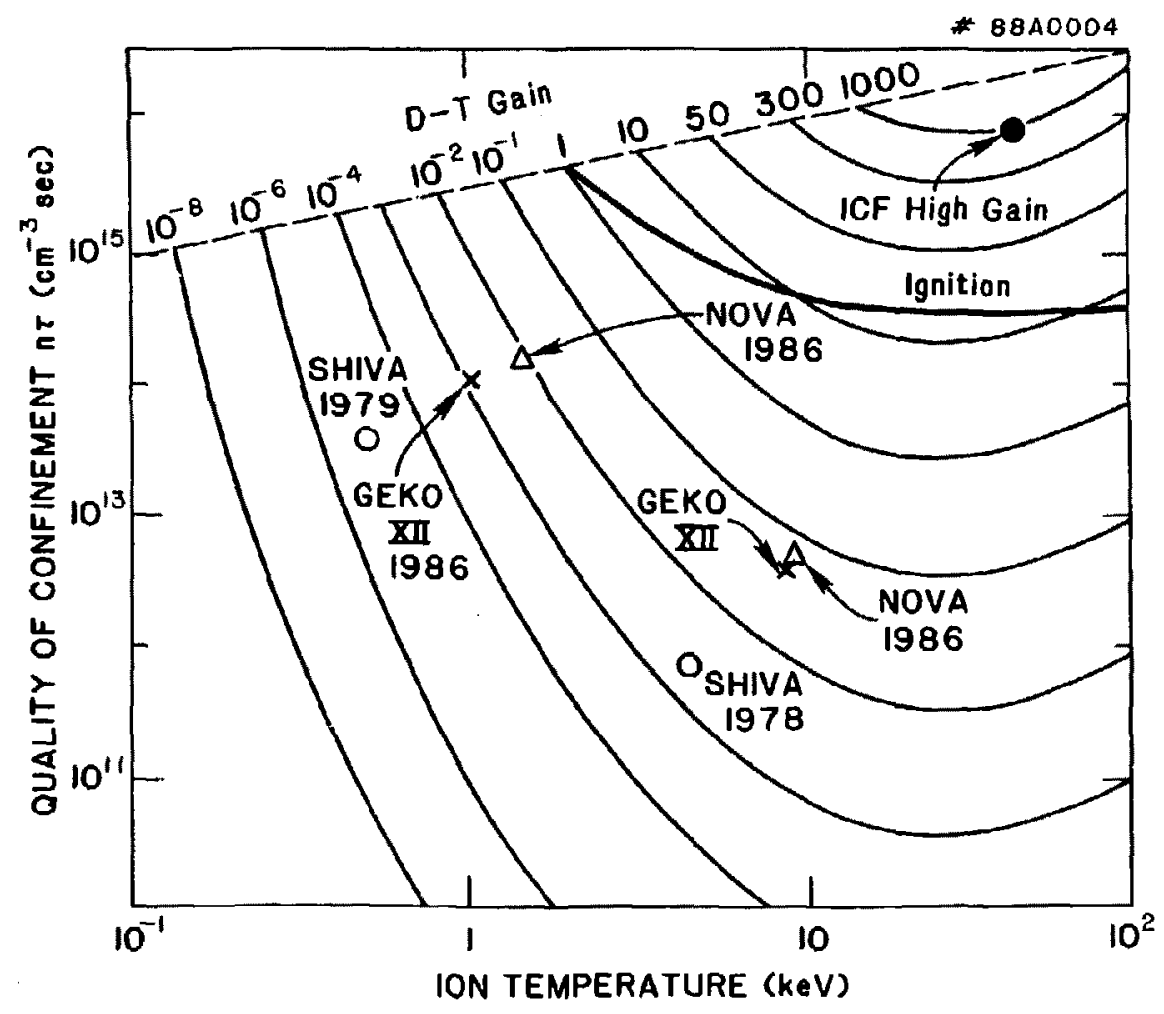

un 


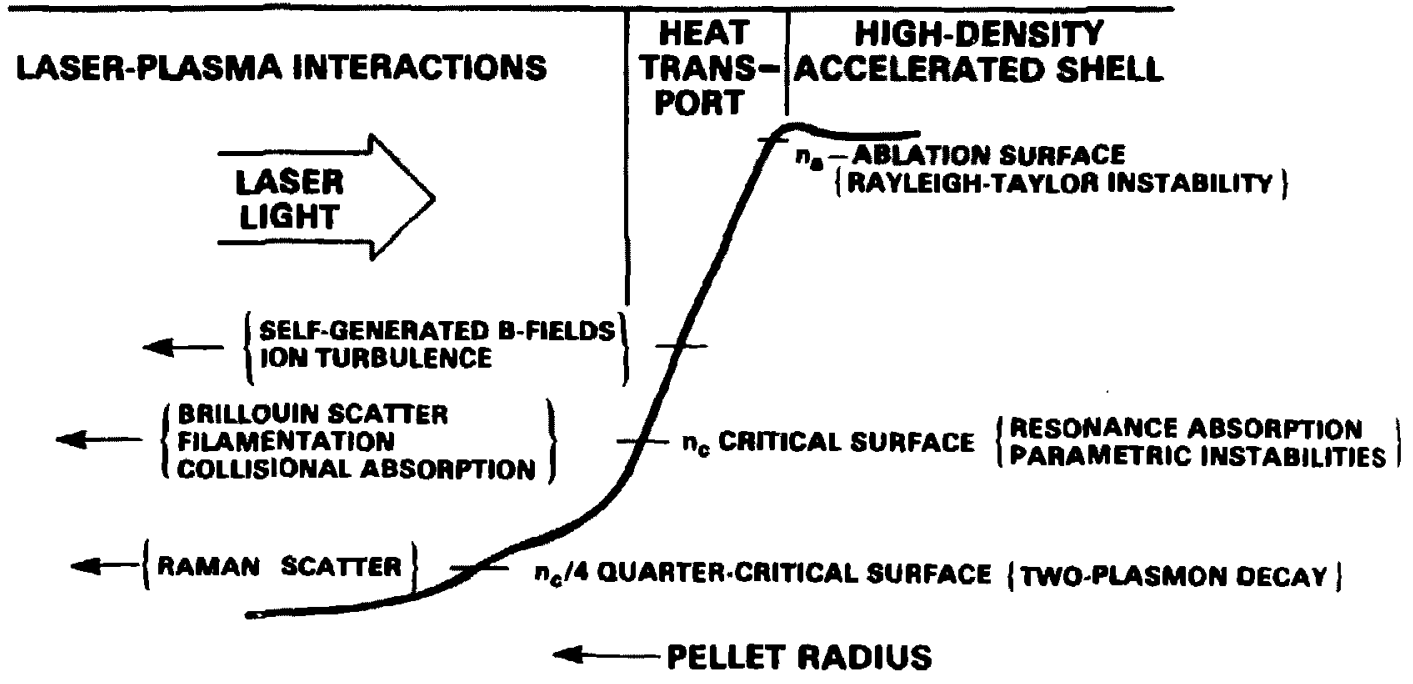

FIg - 11 


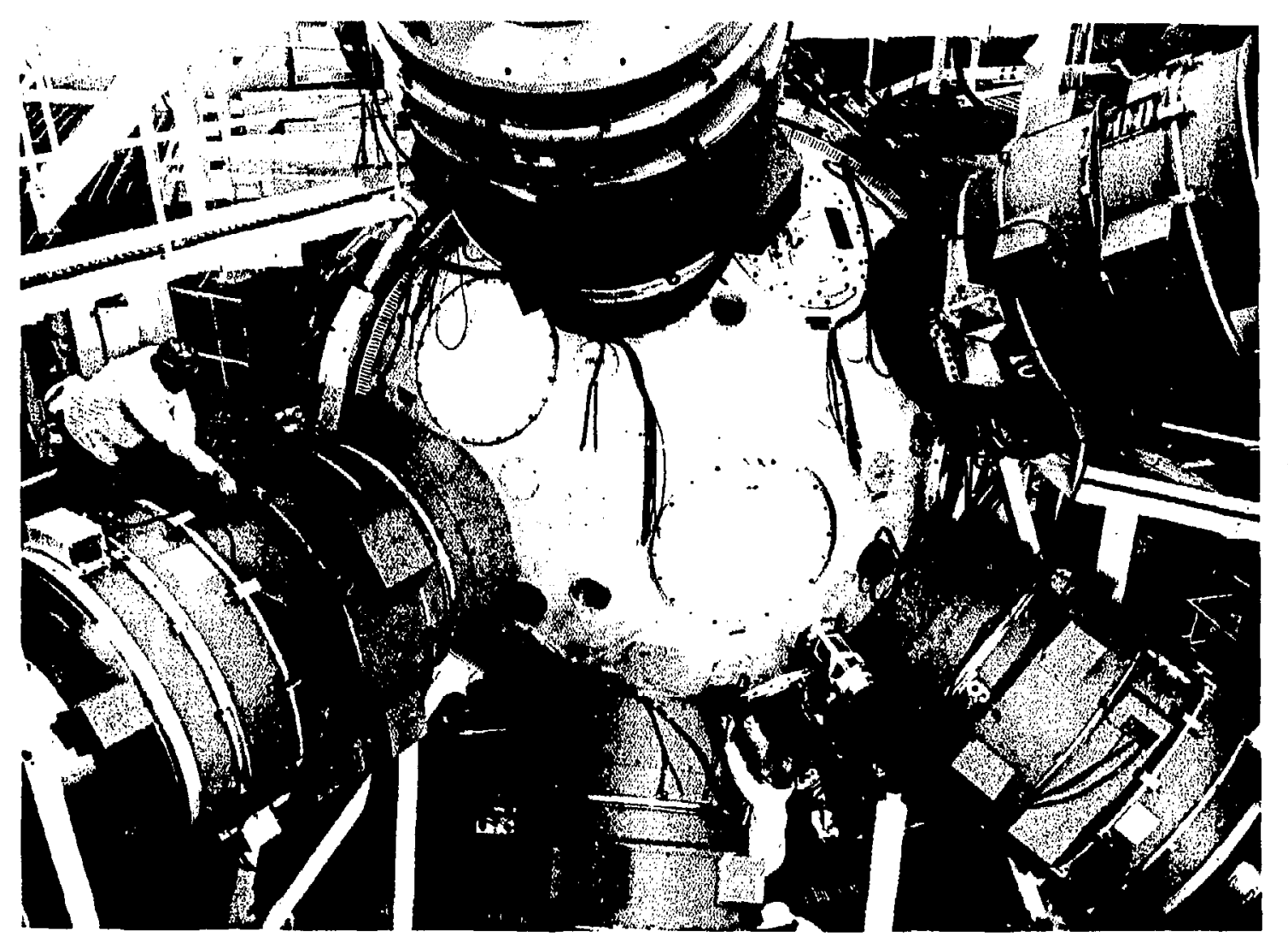

F1g. 12 


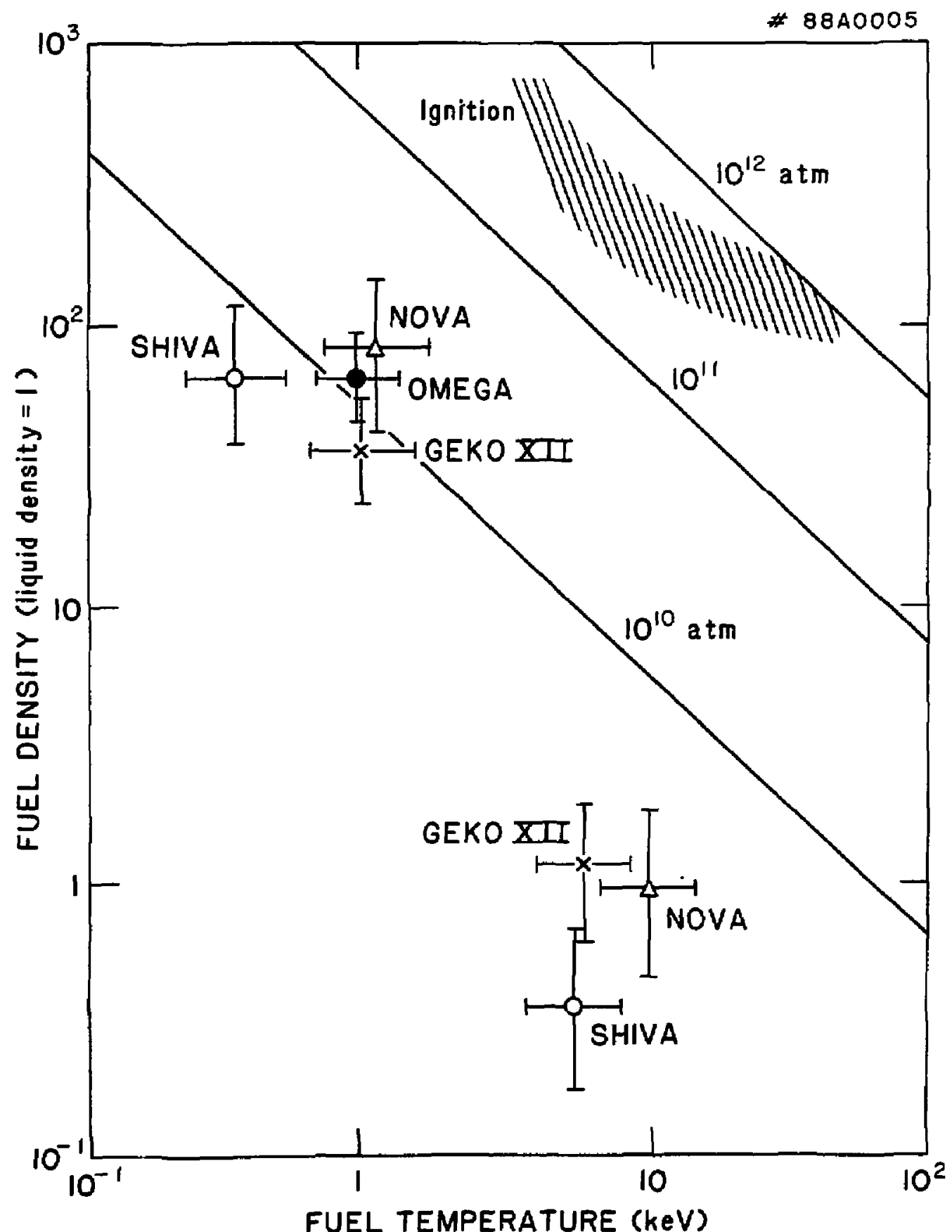

F1g. 13 


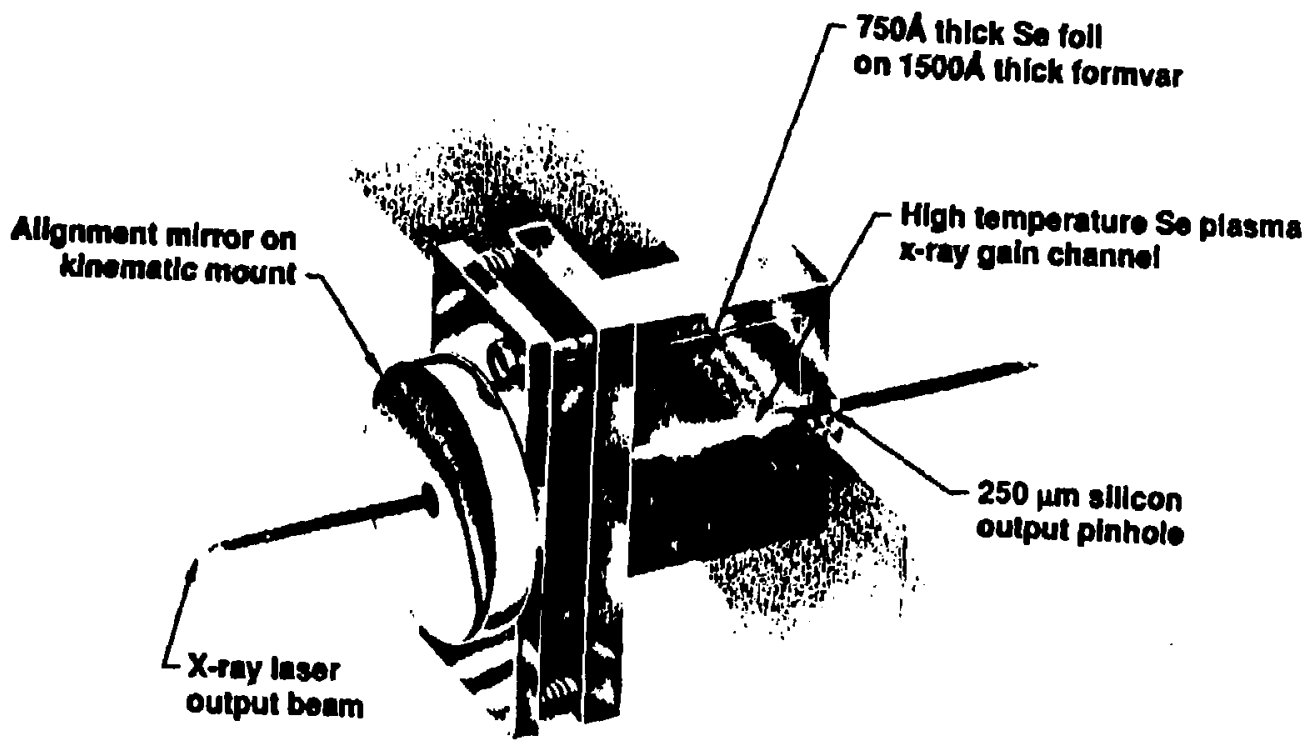

Nova $2 \omega$

oreon laser

10-020205-06790 


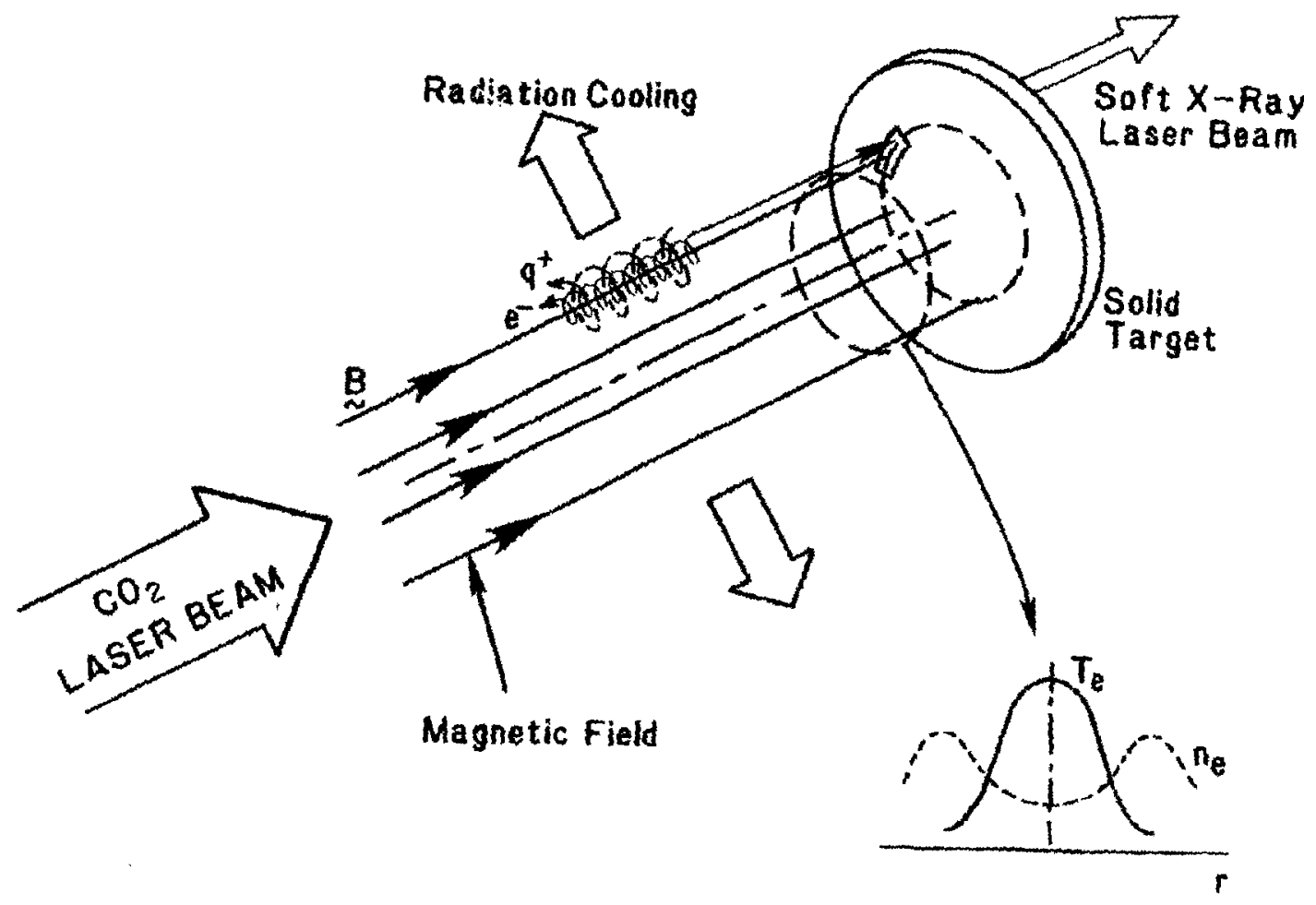


Dr. Frank J. Pootoni, Univ of Wol longong, AUSTRALIA

Prof. M.H. Brennan, Univ Syaney, AuSTRALIA

PIasma Research Lab., Australian Nat. Univ., AUSTRaLIA

Prof, I.R. Jones, FIlinders Univ,., AUSTRALIA

Prof. F. Cap, Inst Theo Phys ${ }_{\lambda}$ AUSTRIA

Prof, M. Helndier, Institut fur Theoretische Physik, AUSTRIA

M. Goossens. Astronomisch Instituut, BELGIUM

Ecole Royale Militaira, Lab de Phys PIasmas, BeLGIuH

Comisston-Eurogan, Dg-XII Fusion Prog, BELGIum

Prof. R. Boucique, Laboratorium voor Natuurkunde, BELGIUA

Dr. P.H. Sakanako, Instituto Fislce, BRAZIL

Instituto De Pesquisas Espaciasi-INPE, BRZIL

Documents office, Atomic Energy of Canoda LIattad, Canpa

Dr. M.P. Bachynski, MPQ Technologies, Inc., Cawnon

Dr. H,M, Skarsgard, Univarsity of Saskatchowan, CAMan

Or. H. Barnard, University of British Columbid, camach

Prof. d. Telchmann, Univ. of Montreal, CAwOA

Prof. S.R. Sreenivasan, Unlyersity of Calgary, CANADA

Prof. Tudor H. Johnston, IMas-Energle, CAwaA

Dr. C.R. Jams, Univ. of Alberta, Canada

Dr. Peter Lukac, Komenskeho Univ, CZECHoslovaKiA

The Librarian, Culha Laboratory. ENELAND

The Librarian, Rutherford Applaton Laboratory, ENGLAND

Mrs. S.A. Hutchinson, JET LIbrary, ENGLANo

c. Nouttet, Lab. de Physique des Mll ieux Ionises, Frarce

5. Rodet, CEN/CADARACHE - Bat 506, fRance

Univ. of loannina, Library of Physics Dapt. GREECE

.Dr. Ton Mual, Academy Blbliographic Ser.., HONG KOv'

Preprint Library. Hungarian Academy of Sciences, Hurcuay

Dr. B. Dasgupta, Saha Inst of Nucl. Phys., INDIA

Dr. P. Kaw, Institute for Plaswa Rostearch, INDIA

Dr. Philip Rosenau, Israel Inst. Tech, ISRAEL

Librarion, Int'l Ctr Theo Phys, ITALy

Prof. G. Rostagni, Univ Di Padova, ITALY

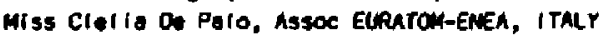

Bibliotece, Instltuto di fisice del Plasmo, ITALY

Dr. H. Yamato, Toshlba Res d Dev, JNPNH

Prof. I. Kowskemi, Atomic Energy Ros. Instituri, JAPAH

Prof. Kyoji Nishikawa, Univ of Hiroshing, JAPAM

Direc. Dept. Lorge Tokadek Ras. JAERI, JAPAN

Prof. Satoshl Itoh, Kyushu UnIvarsity, JAPAY

Resuarch Info Conter, Nagoya University, JAPAN.

Prof. S. Tanaka, Kyoto University, JAPAN

Library, Kyoto University, JAPAN

Prof. Nobuyuki Inoue. University of Tokyo, JAPAN

+\$. Mori, JAERI, JAPAN

Librarian, Korea Advanced Energy Res. Institute, KoReA

Prof. D.1. Chol, Adv. Inst Sel \& Tweh, KoreA

Prof. B.S. Llley, Univarsity of Malkoto, MEU ZEALMD

Institute of Plasen Physles, PEOPLE's RePUalic of CHIM

Librarian, Institute of Phys., PEOPLE'S REPLBLIC of CHIM

Library, Tsing Hu University, PEOPLE'S REPUBLIC OF CHIM
2. Li, Southwest Inst. Physics, PEOPLE's AEPualic of CHINA Prof. JaA.C. Cabral, Inst Superior Tocnico, FORTugal Dr. Octavian Petrus, AL I CUzA University, Romania Dr. Johan de Villlers, Fusion Studies, AEC, SO AFRICA Prof. M.A. Hellberg, University of Natal, SO AFRICA C. L.E.M.A.T., Fusion Div. Library, SPAIN Dr. Lennort Stenflo, University of UMEA, SWEDEN Library, Rayal Inst Toch, SWEDEN

Prof. Hans Wilholnson, Chalners Univ Tech, SWEDEN

Centre Phys des Plasmas, Ecole Palytoch Fed, SWITZERLAND Bibl lotheek, fom-Inst. Yoor PIasma-Fyslca, THE NETHERLANos Or. D.D. Ryutov, Siberlan Acad Sci, USSA

Or. G.A. Ellisaev, Kurchator institute, USSR

Or. V.A. Glukhikh, Inst Electrophyslcal Apparatus, USSR

Or. V.T. Talok, Inst. Phys. Tech, USSR

Or. L.M. Kaurizihnykh, Institute Gan. Physlcs, USSR Nuclear Res. Establi shannt, Julleh Ltd., W. GERuwr Blbliothak, Inst. Fur Plasmaforschung, H. GEPaANY Dr. K. Schindler, Ruhr Univarsitat Bochun, G. GEPWNY ASDEX Resding Rom, IPP/Max-PI anck-I nstitut fur

Pl asmaphys ik, H. GERHAY Librarian, Max-Planck Inetitut, W. GEPawir Prof. R.K. Janev, Inst Phys, YugosLavia 